\title{
Alcohol exposure-induced neurovascular inflammatory priming impacts ischemic stroke and is linked with brain perivascular macrophages
}

\author{
Antoine Drieu, ${ }^{1}$ Anastasia Lanquetin, ${ }^{1}$ Damien Levard, ${ }^{1}$ Martina Clavan, ${ }^{1}$ Francisco Campos, ${ }^{2}$ \\ Aurélien Quenault, ${ }^{1}$ Eloïse Lemarchand, ${ }^{1}$ Mikaël Naveau, ${ }^{3}$ Anne Lise Pitel, ${ }^{4}$ José Castillo, ${ }^{2}$ \\ Denis Vivien,' and Marina Rubio' \\ 1INSERM, Physiopathology and Imaging of Neurological Disorders, UMR-S 1237, Normandie Université, Caen, France. \\ ${ }^{2}$ Clinical Neurosciences Research Laboratory, Health Research Institute of Santiago de Compostela (IDIS), Santiago de \\ Compostela, Spain. ${ }^{3}$ CNRS, UMR-S 3408, GIP Cyceron, Normandie Université, Caen, France. ${ }^{4}$ INSERM, Neuropsychologie et \\ Imagerie de la Mémoire Humaine, UMR-S 1077, Université Paris Sciences et Lettres, Caen, France.
}

\begin{abstract}
Alcohol abuse is a major public health problem worldwide, causing a wide range of preventable morbidity and mortality. In this translational study, we show that heavy drinking (HD) ( $\geq 6$ standard drinks/day) is independently associated with a worse outcome for ischemic stroke patients. To study the underlying mechanisms of this deleterious effect of HD, we performed an extensive analysis of the brain inflammatory responses of mice chronically exposed or not to $10 \%$ alcohol before and after ischemic stroke. Inflammatory responses were analyzed at the parenchymal, perivascular, and vascular levels by using transcriptomic, immunohistochemical, in vivo 2-photon microscopy and molecular MRI analyses. Alcohol-exposed mice show, in the absence of any other insult, a neurovascular inflammatory priming (i.e., an abnormal inflammatory status including an increase in brain perivascular macrophages [PVM]) associated with exacerbated inflammatory responses after a secondary insult (ischemic stroke or LPS challenge). Similar to our clinical data, alcohol-exposed mice showed larger ischemic lesions. We show here that PVM are key players on this aggravating effect of alcohol, since their specific depletion blocks the alcohol-induced aggravation of ischemic lesions. This study opens potentially new therapeutic avenues aiming at blocking alcohol-induced exacerbation of the neurovascular inflammatory responses triggered after ischemic stroke.
\end{abstract}

Conflict of interest: The authors have declared that no conflict of interest exists.

Copyright: () 2020, American Society for Clinical Investigation.

Submitted: March 29, 2019

Accepted: January 15, 2020

Published: February 27, 2020

Reference information: /CI Insight. 2020;5(4):e129226.

https://doi.org/10.1172/jci.

insight.129226.

\section{Introduction}

Alcohol abuse is a major public health problem worldwide, causing a wide range of preventable morbidity and mortality. In the European Union, $89 \%$ of men and $82 \%$ of women are current drinkers; among them, $15.3 \%$ of men and $3.4 \%$ of women are heavy drinkers ( $>6$ drinks/day) (1). In the United States, excessive alcohol use is known to kill about 88,000 people each year, and the cost of excessive alcohol use reached $\$ 249$ billion in 2010 (http://www.cdc.gov/features/costsofdrinking). Alcohol modifies the risk of stroke: light and moderate alcohol consumption ( $0-2$ drinks/day) are associated with a lower risk of ischemic stroke, whereas higher doses of alcohol are associated with an increased risk (2). Importantly, stroke risk associated with high and heavy drinking (HD) in midlife $(<75$ years) predominates over well-known stroke risk factors like hypertension and diabetes (3). However, the impact of alcohol consumption on stroke outcome is less known. Current clinical studies are controversial and have described either an aggravating effect (4) or no effect of HD (5) on stroke severity. Preclinical reports have also described either a protective effect of low alcohol consumption on ischemic stroke $(6,7)$ or larger infarcts in rodents exposed to higher alcohol dose $(6,8)$. However, the mechanisms mediating this aggravation are not well understood. Our previous results on the impact of alcohol consumption on ischemic stroke, obtained in a clinically relevant thromboembolic model of stroke $(9,10)$, have shown that the aggravating effect of excessive drinking is not due to alcohol-induced changes in hemodynamic 
parameters (clot formation, stability, or sensitivity to fibrinolysis) (8). On the other hand, clinical and preclinical data have shown that alcohol consumption may have an impact on inflammation (11-13).

Perivascular macrophages (PVM) are a subpopulation of myeloid cells residing in the CNS. PVM surround brain blood vessels and are located at the perivascular space $(14,15)$. In physiological conditions, PVM have scavenger functions, revealing a role of clearing debris from the CNS (16-18), and can also present antigens to lymphocytes (19). Recent data implicate PVM in several pathological contexts, including brain infections, immune activation, Alzheimer's disease, or multiple sclerosis, suggesting that PVM are a key component of the brain-resident immune system, with broad implications for the pathogenesis of major brain diseases (20). PVM can produce reactive oxygen species and cytokines, linking them to the inflammatory response (21). Due to the major role of inflammation in stroke pathobiology and outcome (22), in this translational study, we aimed at investigating the role of inflammation in the aggravating effect of chronic alcohol drinking on ischemic stroke.

\section{Results}

HD stroke patients show higher stroke baseline severity and worse neurological outcome. A total of 3,645 ischemic stroke patients were included in the retrospective analysis (Figure 1A). HD stroke patients ( $n=424,11.6 \%$; drinking $\geq$ 6 drinks/day in the last 5 years) were significantly younger, were more frequently men, and presented a history of hypertension and higher smoking habits than non-HD stroke patients. In addition, HD stroke patients had more severe ischemic strokes, with a higher infarct volume, higher mortality during hospitalization, worse prognosis at 3 months, and higher levels of markers associated with the inflammatory response. Mortality during hospitalization was significantly increased in $\mathrm{HD}$ stroke patients $(11.7 \%$ vs. $9.7 \%, P<0.0001$; Table 1$)$.

The NIH Stroke Scale (NIHSS) scores at admission, 24 hours, and 48 hours after stroke onset were significantly higher in HD stroke patients (Figure $1 \mathrm{~B}$ and Table 1). Interestingly, significantly more HD stroke patients presented early neurological deterioration (END), defined as the increase of NIHSS in 4 or more points in the first 48 hours after admission (19.2\% vs. 4.4\%; Table 1 and Figure 1B).

$H D$ is independently associated with an increased risk of END. Based on the first univariate analysis, END was used as the main variable to analyze the cohort of stroke patients included in this study (Table 2). In this second univariate analysis, a total 3352 patients were included (3146 without END and 206 with END; 293 fewer patients than for the descriptive analysis, since NIHSS data were not recorded or were lost between the admission and the 48 hours). As expected, patients with END showed significantly higher hemorrhagic transformation, higher infarct volume, and worse prognosis at 3 months. Moreover, patients with END presented higher inflammatory response at admission, characterized by higher leukocyte numbers, as well as C-reactive protein (CRP) levels. Interestingly, significantly more END patients had HD habits $(36.9 \%$ vs. $10.2 \%, P<0.0001$; Table 2$)$.

The inflammatory response is associated with the risk of END in HD stroke patients. We then performed multivariate analysis of END adjusted by HD (Model A, Figure 1C and Supplemental Table 1; supplemental material available online with this article; https://doi.org/10.1172/jci.insight.129226DS1) or by HD and inflammatory markers (Model B, Figure 1D and Supplemental Table 1). Model A showed that HD is independently associated with an increased risk of END (OR $=4.49 ; 95 \%$ CI, 2.94-6.86; $P<0.0001)$ (Figure $1 \mathrm{C}$ and Supplemental Table 1). When inflammatory markers (axillary temperature, leukocyte numbers and CRP) were included in the analysis (Model B, Figure 1D and Supplemental Table 1), the impact of HD on the END OR decreased from 4.49 (obtained on the Model A) to 2.45 (OR $=2.45 ; 95 \%$ CI, $1.01-5.91 ; P<0.0001$ ), showing that the inflammatory response is associated with the risk of END in HD stroke patients. Age and sex do not have a relevant impact on END (Supplemental Table 2).

Finally, we studied the effect of HD on infarct volume. After adjusting for those variables that can interfere with the variable "infarct volume" (age, sex, axillary temperature, leukocyte levels, fibrinogen levels, CRP levels, tissue-type plasminogen activator (tPA) treatment, thrombectomy, hemorrhagic transformation, NIHSS on admission, END, Trial of ORG 10172 in Acute Stroke Treatment (TOAST) classification, and HD; Supplemental Table 3), linear regression analysis of infarct volume showed that the predictor value of $\mathrm{HD}$ was 19.66 (B = 19.66 rate of change; $95 \% \mathrm{CI}, 8.03-31.30 ; P<0.001$; Supplemental Table 4).

HD patients without stroke show high levels of inflammatory markers. Data from the independent cohort of HD patients without stroke showed that the levels of circulating monocytes are significantly increased compared with control healthy subjects $(0.73 \pm 0.27$ vs. $0.2 \pm 0.17$, respectively, $P=0.003$; Table 3$)$. In addition to this, $61.8 \%$ of HD showed increased high-sensitivity CRP (hs-CRP) levels (Table 3 ). 
A

Human stroke cohort (3,645 patients)
C

Model A
END Odds Ratio

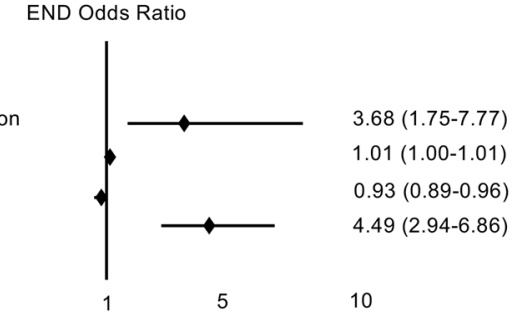

B

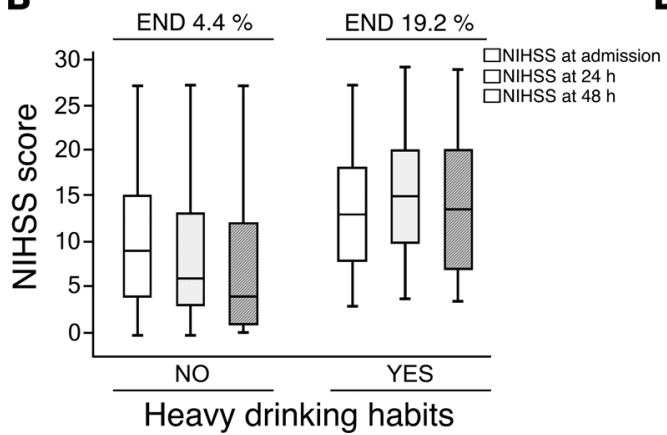

Model B

Hemorrhagic transformation Infarct volume NIHSS on admission Heavy drinking Axillary temperature Leukocytes C-reactive protein $-10$

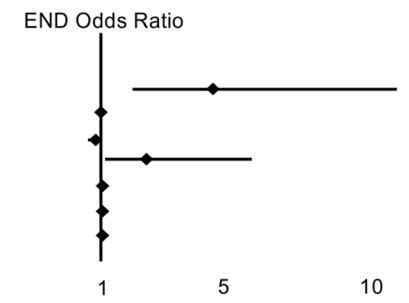

$4.65(2.08-10.41)$ $1.00(1.00-1.00)$ $0.91(0.87-0.95)$ $2.45(1.01-5.91)$ $1.05(0.74-1.50)$ $1.06(0.99-1.13)$ $1.05(1.00-1.08)$

Figure 1. Heavy drinking (HD) aggravates ischemic stroke baseline severity and outcome, and it increases early neurological deterioration (END) risk. Inflammatory markers are independently associated to the alcohol-induced increased risk of END. (A) Schema of the study. (B) Representation of neurological stroke severity determined by the NIH Stroke Scale (NIHSS) at admission and 24 and 48 hours in stroke patients with and without heavy drinking habits. Early neurological deterioration (END) is defined as an increase of NIHSS in 4 or more points in the first 48 hours after admission. (C) Forest plot of the multivariate analysis including variables predicting END $(n=206)$ such as hemorrhagic transformation, infarct volume, NIHSS on admission, and HD habits (Model A). (D) Forest plot of the multivariate analysis of the variables included in the Model A and inflammatory biomarkers (axillary temperature, number of leukocytes levels, and C-reactive protein levels) (Model B).

Alcohol exposure induces a neurovascular inflammatory priming in mice. The first set of experiments aimed to analyze the inflammatory status in the brain of mice drinking $10 \%$ alcohol for 6 weeks $(\sim 5 \mathrm{~g} / \mathrm{kg} /$ day $)$, in the absence of any other insult (Figure 2A). To study microglial cells, brain samples were stained with Iba1, a constitutive marker of microglial cells, and with the lysosomal marker CD68, present in activated microglial cells (23). We found that, whereas the total number of activated microglial (Iba $1^{+} \mathrm{CD} 68^{+}$) cells remained unchanged (Figure 2, B-D), the total area of $\mathrm{CD}^{+} 8^{+}$staining was significantly increased in alcohol-exposed mice ( $P<0.05$ vs. control; Figure 2, $\mathrm{C}$ and $\mathrm{E})$, suggestive of an alcohol-induced increase in microglial activation. Alcohol exposure did not change microglial cell numbers (total number of Iba ${ }^{+}$ cells) (Figure 2, B and F; $n=4$ mice/group). In terms of microglial morphology, alcohol exposure did not change the number of main processes (starting from the soma) (Figure 2, C and G) or the mean area of microglial cells (Figure 2, $\mathrm{C}$ and $\mathrm{H}$ ).

The alcohol-induced increase in microglial activation was demonstrated by an in vivo functional measurement of microglial phagocytosis after the injection of nonionic latex beads in the brain cortex of control and alcohol-exposed mice (Figure 2, I-L). Eight hours after the intracortical injection of the beads, alcohol-exposed mice showed significantly more phagocyted beads (surrounded by Iba1 ${ }^{+}$staining) than control mice (Figure 2 , $\mathrm{I}-\mathrm{K}$ and $\mathrm{M} ; n=4 \mathrm{mice}$ /group; $P<0.05$ vs. control), demonstrating that microglia in alcohol-exposed mice is more prone for phagocytosis. These results show an alcohol-induced microglial priming characterized by a morphological "resting" state - accompanied, however, by an increase in both microglial activation status and phagocytic capacity.

In this study, we also wanted to examine potential inflammatory effects of alcohol exposure on the brain vasculature (Figure 3, A and B). For this reason, we analyzed the endothelial levels of the adhesion molecule P-selectin (CD62P marker), responsible of leukocyte tethering and rolling on the vessel wall. At the vascular level, alcohol-exposed mice showed significantly increased P-selectin ${ }^{+}$blood vessels $(152 \%$ increase; $1.03 \pm 0.31 \mathrm{CD}_{2} \mathrm{P}^{+}$blood vessels $/ \mathrm{mm}^{2} ; n=4$ mice/group; $\mathrm{P}<0.05$ vs. control; Figure $\left.3 \mathrm{~B}\right)$, whereas, in control mice, very low levels of P-selectin $\left(0.31 \pm 0.11 \mathrm{CD}^{2} \mathrm{P}^{+}\right.$blood vessels $\left./ \mathrm{mm}^{2}\right)$ were detected, demonstrating an alcohol-induced endothelial activation in the absence of any other brain injury. 
Table 1. Univariate analysis of stroke patients with and without heavy drinking habits (>6 standard drinks/day)

\begin{tabular}{|c|c|c|c|}
\hline \multicolumn{3}{|c|}{ Heavy drinking } & \\
\hline & $\begin{array}{c}\text { No } \\
n=3221\end{array}$ & $\begin{array}{c}\text { Yes } \\
n=424\end{array}$ & \\
\hline Age (years) & $72.60 \pm 14.06$ & $66.79 \pm 11.87$ & $<0.0001$ \\
\hline Men (\%) & 50.5 & 85.8 & $<0.0001$ \\
\hline History of hypertension (\%) & 64.6 & 52.4 & $<0.0001$ \\
\hline History of diabetes (\%) & 24.7 & 22.6 & 0.368 \\
\hline History of ischemic heart disease (\%) & 11.5 & 11.3 & 0.491 \\
\hline Axillary temperature at admission $\left({ }^{\circ} \mathrm{C}\right)$ & $36.3 \pm 0.6$ & $36.6 \pm 0.6$ & $<0.0001$ \\
\hline Leukocytes on admission $\left(\times 10^{3} / \mathrm{mL}\right)$ & $8.8 \pm 3.1$ & $9.9 \pm 3.2$ & 0.135 \\
\hline Fibrinogen on admission (mg/dL) & $416.9 \pm 97.6$ & $439.5 \pm 87.1$ & $<0.0001$ \\
\hline C-reactive protein on admission (mg/L) & $2.7 \pm 4.2$ & $3.5 \pm 3.1$ & $<0.0001$ \\
\hline Infarct volume (mL), $n=2121$ & $40.1 \pm 70.4$ & $46.9 \pm 64.8$ & 0.021 \\
\hline NIHSS on admission, $n=3645$ & 9 (5-15 NIHSS) & 14 (11-19 NIHSS) & $<0.0001$ \\
\hline NIHSS at 24 hours, $n=3277$ & 6 (3-10 NIHSS) & 18 (9-20 NIHSS) & $<0.0001$ \\
\hline NIHSS at 48 hours, $n=3352$ & 4 (1-10 NIHSS) & 14 (7-20 NIHSS) & $<0.0001$ \\
\hline $\operatorname{END}(\%), n=3352$ & 4.4 & 19.2 & $<0.0001$ \\
\hline mRS at 3 months, $n=2935$ & 2 (1-4 mRS) & 3 (2-4 mRS) & $<0.0001$ \\
\hline Poor outcome at 3 months (\%), $n=2935$ & 53.6 & 65.1 & $<0.0001$ \\
\hline \multicolumn{2}{|l|}{ TOAST } & & 0.286 \\
\hline Atherothrombotic (\%) & 23.2 & 26.7 & \\
\hline Cardioembolic (\%) & 37.1 & 32.8 & \\
\hline Small vessel disease (\%) & 7.3 & 6.1 & \\
\hline
\end{tabular}

To determine the functional impact of the increase in P-selectin expression, we performed in vivo 2-photon microscopy analyses to measure venular leukocyte adhesion and rolling in mice exposed or not to alcohol, after the i.v. injection of FITC-Dextran (allowing blood vessels visualization) and Rhodamine-6G (staining leukocytes). Alcohol-exposed mice showed significantly more adherent (Figure 3, C and D) and rolling (Figure 3, E and F) leukocytes compared with control mice (317\% and $724 \%$ increase, respectively; $n=6$ mice/group; $P<0.05$ vs. control).

Concerning mRNA expression of inflammatory markers, alcohol-exposed mice showed a significant increase in TGF- $\beta$ mRNA levels ( $P<0.05 ; n=6$ mice/group) compared with control mice that was not accompanied by changes in proinflammatory cytokines such as IL-1 $\beta$, TNF, IL-6, P-selectin, TLR4, or VCAM1 (Table 4).

We also studied blood-brain barrier (BBB) integrity after alcohol exposure by using 3 different methods. Our results showed that chronic alcohol exposure does not seem to alter BBB integrity, since neither Evans Blue extravasation measured by near-infrared fluorescence (NIRF) imaging (Supplemental Figure 1, A-D; $n=4$ mice/group), positive signals of gadolinium extravasation measured by T1-weighted (T1-w) MRI (Supplemental Figure 1, E-H; $n=4$ mice/group) or fibrinogen deposits (Supplemental Figure 1I; $n=4$ mice/group) were found in alcohol-exposed mice.

Concerning the potential alcohol-induced neuronal death, we did not find any fluoro-jade C-positive staining in alcohol-exposed mice (Supplemental Figure 1B; $n=4$ mice/group). 
Table 2. Univariate analysis of stroke patients with and without early neurological deterioration

\begin{tabular}{|c|c|c|c|}
\hline \multicolumn{4}{|c|}{ Early neurological deterioration } \\
\hline & $\begin{array}{c}\text { No } \\
n=3146\end{array}$ & $\begin{array}{c}\text { Yes } \\
n=206\end{array}$ & \\
\hline Age (years) & $72.1 \pm 13.4$ & $73.8 \pm 11.9$ & 0.977 \\
\hline Men (\%) & 54.7 & 59.2 & 0.219 \\
\hline History of hypertension (\%) & 62.8 & 62.6 & 0.509 \\
\hline History of diabetes (\%) & 24.5 & 23.3 & 0.384 \\
\hline $\begin{array}{l}\text { History of ischemic heart disease } \\
\text { (\%) }\end{array}$ & 11.3 & 14.1 & 0.135 \\
\hline $\begin{array}{l}\text { Axillary temperature at admission } \\
\left({ }^{\circ} \mathrm{C}\right)\end{array}$ & $36.3 \pm 0.6$ & $36.4 \pm 0.8$ & 0.005 \\
\hline Leukocytes on admission $\left(\times 10^{3} / \mathrm{mL}\right)$ & $8.9 \pm 3.3$ & $10.4 \pm 4.2$ & 0.019 \\
\hline Fibrinogen on admission (mg/dL) & $426.7 \pm 102.8$ & $422.8 \pm 120.5$ & 0.255 \\
\hline tPA treatment (\%) & 23.5 & 22.3 & 0.389 \\
\hline Trombectomy (\%) & 2.7 & 3.9 & 0.210 \\
\hline Hemorrhagic transformation (\%) & 9.4 & 17.1 & $<0.0001$ \\
\hline Infarct volume (mL) & $50.3 \pm 77.1$ & $107.7 \pm 107.8$ & $<0.0001$ \\
\hline NIHSS at admission & 12 (NIHSS 7-17) & 13 (NIHSS 8-15) & 0.017 \\
\hline mRS at 3 months & 3 (mRS 1-4) & 5 (mRS 3-6) & $<0.0001$ \\
\hline Poor outcome at 3 months (\%) & 51.0 & 81.2 & $<0.0001$ \\
\hline TOAST & & & 0.613 \\
\hline Atherothrombotic (\%) & 24.3 & 25.2 & \\
\hline Cardioembolic (\%) & 35.8 & 38.3 & \\
\hline Small vessel disease (\%) & 7.2 & 4.4 & \\
\hline
\end{tabular}

Alcohol exposure exacerbates brain neurovascular inflammatory reactions after an acute systemic insult. Alcohol-exposed mice showed a global exacerbated inflammatory response in the brain 24 hours after an acute i.p. injection of LPS compared with control mice (Figure 4A). At the parenchymal level, alcohol-exposed mice receiving LPS showed a significant increase in (a) total microglial cells (Iba1 ${ }^{+}$cells) (Figure 4, B and C; $n=5$ mice/group; $P<0.05$ vs. control), (b) activated microglia (Iba1 ${ }^{+} \mathrm{CD} 68^{+}$cells) (Figure 4, B and D; $n=5$ mice/group; $P<0.05$ vs. control), and (c) $\mathrm{CD}^{+} 8^{+} \mathrm{Iba1}^{-}$cells that we considered as macrophages (Figure 4, B and E) compared with LPS-injected control mice.

At the vascular level, P-selectin immunostaining (Figure 4, F and G) was significantly increased in alcohol-exposed mice receiving LPS compared with control mice ( $n=5$ mice/group; $P<0.05$ vs. control). Molecular MRI also showed a significantly increased number of P-selectin-coupled microsized particles of iron oxide-positive $\left(\mathrm{MPIO}^{+}\right.$) blood vessels in alcohol-exposed mice receiving LPS (Figure $4, \mathrm{H}$ and $\mathrm{I} ; n=5$ mice/group; $P<0.05$ vs. control). Consequently, venular leukocyte adhesion (Figure $4, \mathrm{~J}$ and $\mathrm{K}$ ) and rolling (Figure 4, L and M), measured by intravital 2-photon microscopy, were significantly increased in alcohol-exposed mice receiving LPS compared with control mice $(n=5$ mice/ group; $P<0.05$ vs. control).

Alcohol exposure aggravates stroke lesions and exacerbates neurovascular inflammatory responses after ischemic stroke in mice. Alcohol-exposed mice showed significantly larger ischemic lesions than control mice (drinking only water) 24 hours after stroke onset (Figure 5, A-C; $n=8$ mice/group; $P<0.05$ vs. control) (8). These data on lesion volume are part of a previously published figure (8). No hemorrhagic transformation was detected in any mice (data not shown). 
Table 3. Heavy drinkers have higher levels of inflammatory markers, in the absence of any other injury

\begin{tabular}{|c|c|c|c|}
\hline & $\begin{array}{c}\text { Control subjects } \\
\qquad n=\mathbf{2 1}\end{array}$ & $\begin{array}{c}\text { Heavy drinkers } \\
n=\mathbf{3 4}\end{array}$ & $\boldsymbol{P}$ \\
\hline Age & $\begin{array}{c}43.8 \pm 7.4 \\
\text { (29-55 years) }\end{array}$ & $\begin{array}{c}45.7 \pm 9.2 \\
\text { (33-66 years) }\end{array}$ & 0.29 \\
\hline BMI & $\begin{array}{c}25.3 \pm 4.4^{\mathrm{A}} \\
(19.5-35.5 \mathrm{BMI})\end{array}$ & $\begin{array}{c}23.9 \pm 4.4 \\
(17.3-39.8 \mathrm{BMI})\end{array}$ & 0.32 \\
\hline AUDIT & $\begin{array}{c}2.9 \pm 1.6 \\
\text { (scores } 0-6 \text { ) }\end{array}$ & $\begin{array}{c}28.9 \pm 7.7 \\
\text { (scores } 4-40)\end{array}$ & $<0.001$ \\
\hline Leucocytes (g/L) & $\begin{array}{c}6.4 \pm 1.7 \\
(3.7-9.7 \mathrm{~g} / \mathrm{L})\end{array}$ & $\begin{array}{c}7.0 \pm 2.1 \\
(3.5-12.3 \mathrm{~g} / \mathrm{L})\end{array}$ & 0.24 \\
\hline Neutrophils (g/L) & $\begin{array}{c}3.6 \pm 1.4 \\
(1.7-7.2 \mathrm{~g} / \mathrm{L})\end{array}$ & $\begin{array}{c}4.1 \pm 1.7 \\
(1.7-8.6 \mathrm{~g} / \mathrm{L})\end{array}$ & 0.28 \\
\hline Eosinophils (g/L) & $\begin{array}{c}0.17 \pm 0.08 \\
(0.04-0.31 \mathrm{~g} / \mathrm{L})\end{array}$ & $\begin{array}{c}0.18 \pm 0.13 \\
(0-0.53 \mathrm{~g} / \mathrm{L})\end{array}$ & 0.80 \\
\hline Monocytes (g/L) & $\begin{array}{c}0.52 \pm 0.17 \\
(0.27-0.99 \mathrm{~g} / \mathrm{L})\end{array}$ & $\begin{array}{c}0.73 \pm 0.27 \\
(0.11-1.42 \mathrm{~g} / \mathrm{L})\end{array}$ & 0.003 \\
\hline C Reactive protein $>3 \mathrm{mg} / \mathrm{L}$ & & $61.8 \%(21$ of 34$)$ & \\
\hline \multicolumn{4}{|c|}{$\begin{array}{l}\text { Demographic and clinical characteristics, leukocyte cell counting, and C-reactive Protein (CRP) levels in an independent cohort of healthy control } \\
\text { participants and heavy drinkers recruited in the Addiction Unit of the University Hospital of Caen (Normandy, France) while they were receiving withdrawal } \\
\text { treatment as inpatients. Data are expressed as mean } \pm \text { SD; parenthesis data correspond to interval. AUDIT, Alcohol Use Disorders Identification Test. } \\
{ }^{A} \text { Missing data. } N=20 \text {. }\end{array}$} \\
\hline
\end{tabular}

Microglial/macrophage reaction after stroke (Figure 5, D-K) was exacerbated in the ipsilateral cortex of alcohol-exposed mice, with significant increases in (a) the total number of $\mathrm{Iba}^{+}{ }^{+}$cells in the peri-infarct area (Figure 5F; $n=3$ mice/group; $P<0.05$ vs. control), (b) the number of $\mathrm{CD} 68^{+} \mathrm{Iba} 1^{+}$cells in the peri-infarct area and the ischemic core (Figure 5G; $n=3$ mice/group; $P<0.05$ vs. control), and (c) the area of lysosomal CD68 staining in the ipsilateral hemisphere of alcohol-exposed mice compared with control (Figure $5 \mathrm{H} ; n=3$ mice/group; $P<0.05$ vs. control). The number of processes starting from the soma was significantly decreased in the ipsilateral cortex of alcohol-exposed mice compared with control mice (Figure 5I; $n=3$ mice/group; $P<0.05$ vs. control), suggestive of an increased microglial phagocytic phenotype

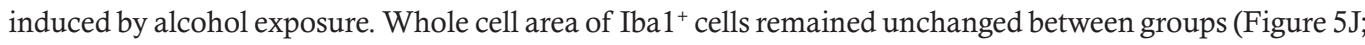
$n=3$ mice/group). The number of $\mathrm{CD}^{+} 8^{+} \mathrm{Iba1}^{-}$cells was significantly increased in the peri-infarct area of alcohol-exposed mice 24 hours after stroke onset (Figure $5 \mathrm{~K} ; n=3$ mice/group; $P<0.05$ vs. control).

Vascular activation was exacerbated in alcohol-exposed mice after stroke (Figure 6, A-F). The area of P-selectin in blood vessels was significantly increased in alcohol-exposed mice 24 hours after stroke onset (Figure 6C; $n=3$ mice/group; $P<0.05$ vs. control), although the number of $\mathrm{P}$-selectin ${ }^{+}$blood vessels showed no changes between groups (Figure 6B). Similarly, molecular MRI (Figure 6D) showed significantly increased hyposignals corresponding to P-selectin-coupled MPIOs adhering to blood vessels in the ipsilateral cortex of alcohol-exposed mice 24 hours after stroke onset (Figure $6 \mathrm{E} ; n=5-6$ mice/group; $P<0.05$ vs. control), with no changes in the total number of P-selectin $\mathrm{MPIO}^{+}$blood vessels (Figure $6 \mathrm{~F}$ ).

Venular leukocyte adhesion (Figure 6, H and I) and rolling (Figure 6, J and K) were both significantly increased in alcohol-exposed mice compared with control mice (317\% and $724 \%$ increase, respectively; $n=6$ mice/group; $P<0.05$ vs. control) 24 hours after stroke. Schemas in Figure $6 \mathrm{G}$ show the relative position of the middle cerebral artery (MCA) occlusion site and the thinned-skull cranial window where in vivo 2-photon imaging was performed.

mRNA levels of IL-1 $\beta$, P-selectin, and TNF were significantly increased in alcohol-exposed mice in the ipsilateral cortex 24 hours after stroke onset compared with control mice $(P<0.05 ; n=6$ mice/group; Table 4).

PVM depletion prevents the aggravating effect of chronic alcohol consumption on ischemic stroke. In addition to the neurovascular priming profile found in alcohol-exposed mice, we also detected a significant increase in 
A

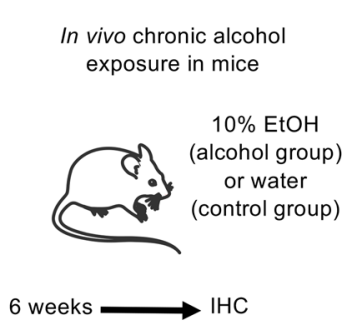

D

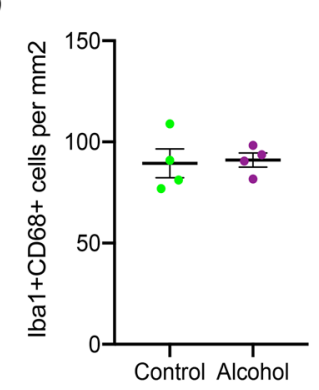

I

In vivo microglial phagocytic capacity

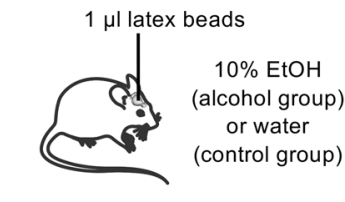

8 hours $\longrightarrow \mathrm{IHC}$
B

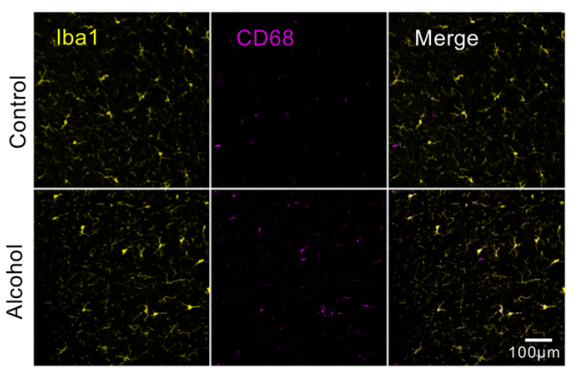

E

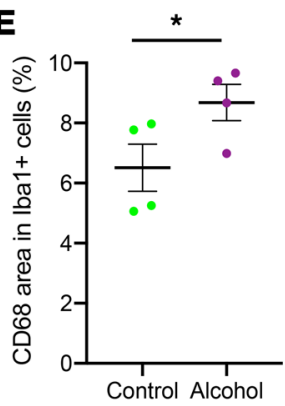

$\mathbf{J}$

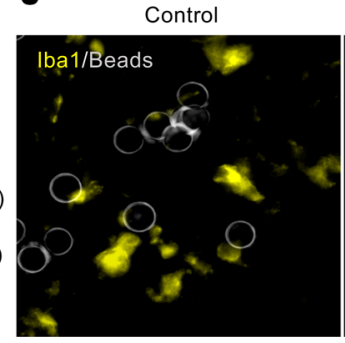

$\mathbf{F}$

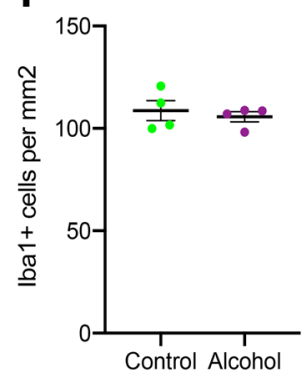

C

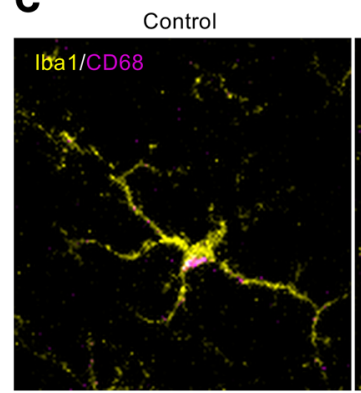

G
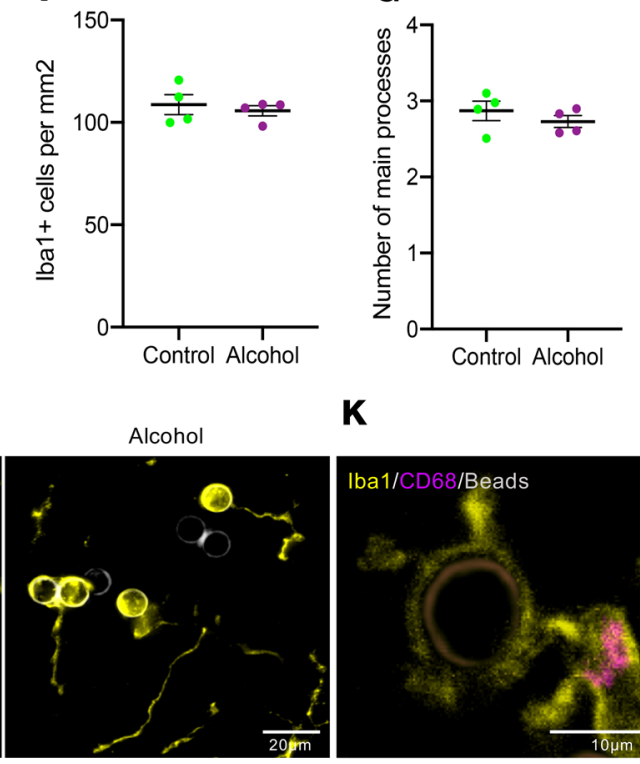

$\mathbf{K}$

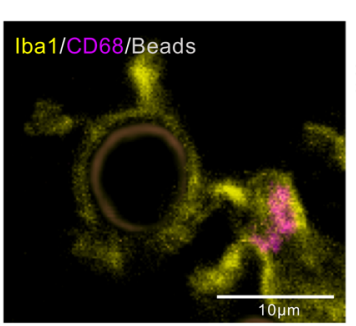

Alcohol

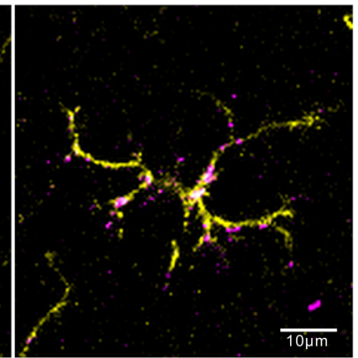

H
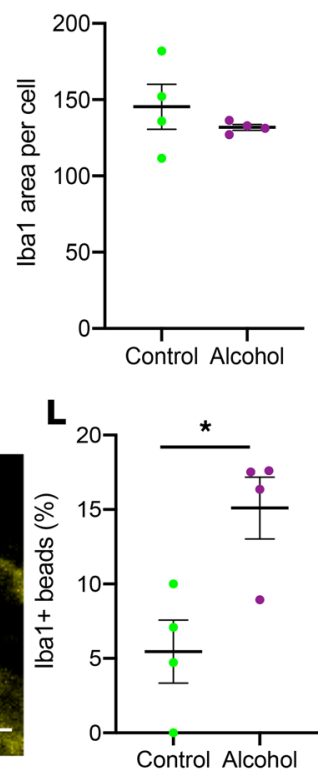

M

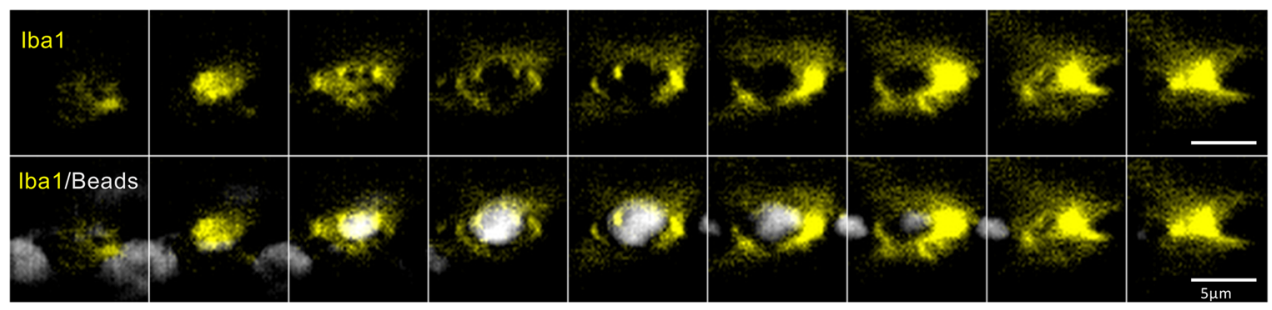

Figure 2. Alcohol exposure provokes a microglial priming in mice. (A) Experimental design to study the effects of chronic alcohol exposure on microglia. (B) Representative photomicrographs of microglial cells stained with lba1 and CD68. Scale bar: $100 \mu$ m. (C) High-magnification representative photomicrographs of microglial cells in control and alcohol-exposed mice. Scale bar: $10 \mu \mathrm{m}$. (D) Quantification of Iba1 ${ }^{+}$CD68 ${ }^{+}$cells. (E) Quantification of CD68 area on Iba1 ${ }^{+}$microglial cells. (F) Quantification of Iba1+ cells. (G) Quantification of the number of processes starting from the soma. (H) Quantification of the area of microglial cells. $n=4$ mice per group. (I) Experimental design to in vivo study the microglial phagocytic capacity: $1 \mu \mathrm{L}$ of latex beads were injected in the cortex of control and alcohol-exposed mice. Eight hours later, phagocytosed latex beads were quantified by immunohistochemical analyses. (J) Representative photomicrographs of Iba1+ microglial cells and latex beads. Scale bar: 20 $\mu \mathrm{m}$. (K) Detail of a latex bead phagocytosed by a microglial cell in an alcohol-exposed mouse. Note the lysosomal activation (CD68, red) at the apex of microglial process. Scale bar: $10 \mu \mathrm{m}$. (L) Quantification of phagocytosed latex beads/total number of beads. $n=4$ mice per group; ${ }^{*} P<0.05$ versus Control, Mann-Whitney $U$ test.

(M) Sequential confocal photomicrographs of a phagocytosed latex bead.

the number of brain PVM (Figure 7, A-C; $n=4$ mice/group; $P<0.05$ vs. control). PVM are a subpopulation of resident brain macrophages located at the perivascular space (Supplemental Figures 2, A-E). In this study, we have measured only submeningeal signals on IHC and in vivo imaging experiments to focus on PVM and no other border-associated macrophages. In addition to their specific shape and location surrounding brain blood vessels (Supplemental Figure 2), in naive conditions, PVM can be distinguished from microglia since they are very low positive for Iba1. PVM can be stained with both CD68 and CD206 (Supplemental Figure 2 and Figure 7, B and C). Concerning the origin of the increased number of PVM 
A

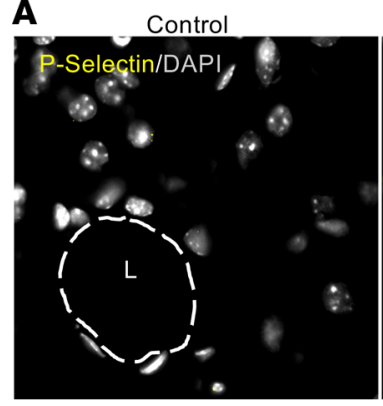

D

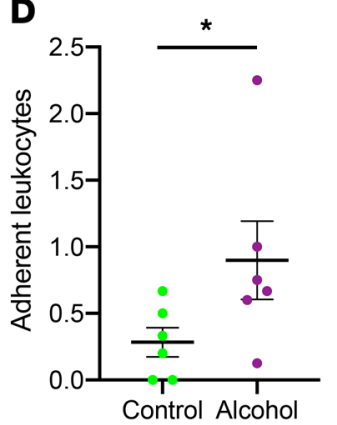

Alcohol

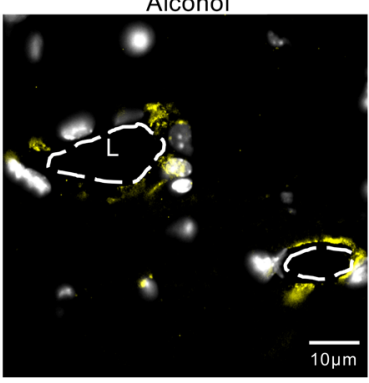

E

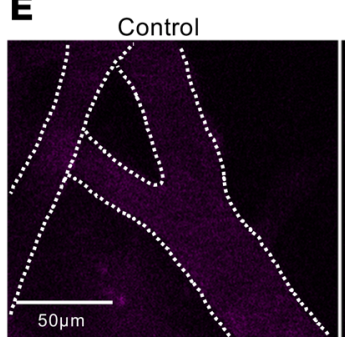

B
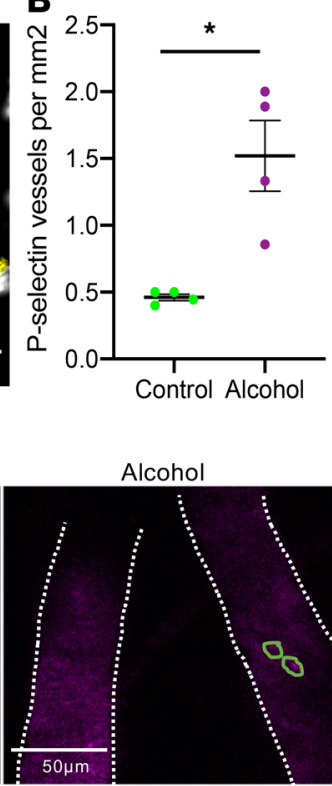

C

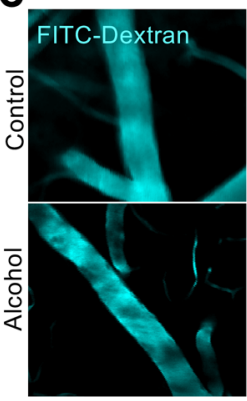

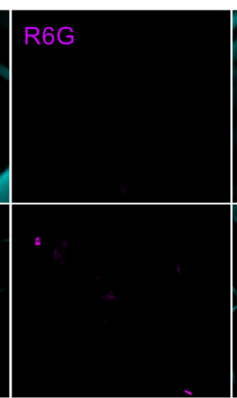

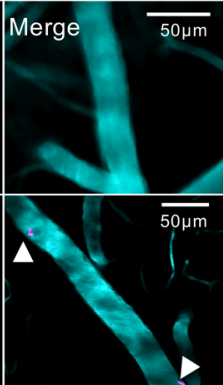

$\mathbf{F}$

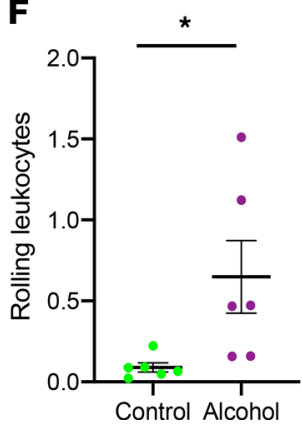

Figure 3. Alcohol exposure provokes endothelial activation in the brain. (A) Representative photomicrographs of P-selectin ${ }^{+}$vessels in control and alcohol-exposed mice (note the absence of positive staining in control mice). Dotted lines represent the lumen (L) of the blood vessel. Scale bar: $10 \mu \mathrm{m}$. (B) Quantification of P-selectin signal in the brain cortex of control and alcohol-exposed mice. $n=4$ mice per group. (C) Compilation of in vivo time-lapse images obtained by 2-photon microscopy showing Rhodamine-6G (R6G)+ leukocyte adhesion (arrowheads) in control and alcohol-exposed mice (note the absence of R6G+ cells in control mice). Scale bars: $50 \mu \mathrm{m}$. (D) Quantification of adherent leukocytes. (E) Representative time-lapse images of leukocyte rolling (green circles). Scale bars: $50 \mu \mathrm{m}$. (F) Quantification of rolling leukocytes per second. $n=4$ mice per group. ${ }^{*} P<0.05$ versus control, Mann-Whitney $U$ test.

after alcohol exposure, the longitudinal study of peripheral macrophage accumulation in the brain suggest the absence of a marked peripheral PVM recruitment (Supplemental Figure 3, A-F). Proliferation measurements by Ki67 staining were performed in harvested brains at the end of the alcohol exposure and have not shown positive signals of PVM proliferation (Supplemental Figure 3, G and H), perhaps because the measurements have been done at a single time point at the end of the alcohol exposure period.

To determine the specific role of brain PVM on ischemic stroke outcome in both healthy (naive) and primed mice (chronically exposed to alcohol), we depleted PVM by injecting clodronate-encapsulated (CLO-encapsulated) liposomes to mice exposed or not to 10\% ethanol for 6 weeks (Figure 7D); control mice received PBS-encapsulated liposomes. In the adult brain, the i.c.v. injection of CLO depletes PVM (as well as meningeal macrophages) without affecting microglial cells or peripheral mononuclear cells (21), except for a transient reduction in the number of Kupffer cells in the liver (24). In our study, we have observed that the i.c.v. injection of CLO efficiently depletes PVM, but also provokes a series of inflammatory responses per se. Our data show that CLO acutely induced, per se, an inflammatory response in the ventricle area at 2 days after injection, which was not visible anymore at 6 days by molecular MRI imaging (P-selectin-coupled MPIOs) (Supplemental Figure 4, A and B; $n=4$ mice/group). Our IHC data, focused on the injection site (the ventricle area), confirmed the data obtained by MRI and showed the absence of P-selectin ${ }^{+}$staining (Supplemental Figure 4C), as well as no neutrophil infiltration (Ly6G ${ }^{+}$staining) at that time (Supplemental Figure 4D). Microglia was not affected by the injection of CLO (Supplemental Figure 4D; $n=4$ mice/group).

Flow cytometry experiments were performed at 5 days after injection, to respect the same timing profile as the one used in the stroke study. Our data show that there were no differences in the numbers of $\operatorname{CD} 45^{10} /$ $\mathrm{CD}_{11 b^{+}}$cells (considered as resting microglia; Supplemental Figure $5 \mathrm{~B}$ ), CD45 $\mathrm{hi} / \mathrm{CD} 11 \mathrm{~b}^{+}$cells (considered as infiltrated macrophages; Supplemental Figure 5C), CD11 $\mathrm{c}^{+} \mathrm{CD} 11 \mathrm{~b}^{+}$cells (considered as DCs; Supplemental Figure 5E), or Ly6 $\mathrm{G}^{+}$cells (considered as granulocytes; Supplemental Figure 5F) between PBS- and CLO-treated mice. However, the numbers of $\mathrm{CD} 45^{\text {int }} \mathrm{Cd} 11 \mathrm{~b}^{+}$cells (considered as activated microglia; Supplemental Figure 5D), $\mathrm{CD}^{+}$(considered as total lymphocytes; Supplemental Figure 5G), $\mathrm{CD}^{+}$(Supplemental Figure $5 \mathrm{H}$ ), and $\mathrm{CD}^{+}$(Supplemental Figure 5I) lymphocytes were increased in CLO-treated mice 5 days after CLO injection. 
Table 4. Alcohol exposure alters the gene expression of inflammatory markers in the brain of mice, both in the absence of any other insult and after ischemic stroke

\begin{tabular}{|c|c|c|c|c|c|c|c|c|c|}
\hline & & & IL-1b & IL-6 & P-selectin & TGF- $\beta$ & TLR4 & TNF & VCAM1 \\
\hline \multirow{2}{*}{\multicolumn{2}{|c|}{ Basal }} & C & $0.19 \pm 0.05$ & $0.67 \pm 0.28$ & $1.89 \pm 1.38$ & $0.69 \pm 0.11$ & $0.80 \pm 0.20$ & $0.44 \pm 0.18$ & $0.78 \pm 0.15$ \\
\hline & & A & $0.54 \pm 0.22$ & $1.25 \pm 0.33$ & $0.44 \pm 0.14$ & $1.00 \pm 0.14^{A}$ & $1.27 \pm 0.24$ & $0.19 \pm 0.01$ & $1.02 \pm 0.21$ \\
\hline \multirow{4}{*}{$\begin{array}{l}\text { MCAo + } 24 \\
\text { hours }\end{array}$} & \multirow{2}{*}{ Contro } & C & $0.14 \pm 0.03$ & $0.25 \pm 0.07$ & $0.22 \pm 0.06$ & $0.99 \pm 0.20$ & $0.77 \pm 0.18$ & $0.06 \pm 0.02$ & $0.97 \pm 0.24$ \\
\hline & & A & $0.15 \pm 0.05$ & $0.11 \pm 0.03$ & $0.13 \pm 0.07$ & $0.82 \pm 0.14$ & $0.64 \pm 0.12$ & $0.08 \pm 0.00$ & $0.73 \pm 0.13$ \\
\hline & \multirow{2}{*}{ Ipsi } & C & $3.01 \pm 0.99$ & $14.00 \pm 4.04$ & $9.32 \pm 1.93$ & $1.90 \pm 0.17$ & $1.77 \pm 0.28$ & $1.45 \pm 0.23$ & $1.39 \pm 0.21$ \\
\hline & & A & $5.51 \pm 0.94^{A}$ & $20.54 \pm 3.11$ & $17.52 \pm 2.21^{A}$ & $2.17 \pm 0.26$ & $2.31 \pm 0.21$ & $2.51 \pm 0.38^{A}$ & $1.29 \pm 0.22$ \\
\hline
\end{tabular}

Gene expression was calculated using 2 housekeeping genes. The increase of the inflammatory markers expression was exacerbated in alcoholexposed mice after thromboembolic stroke (basal, $n=4$ mice per group; MCAo +24 hours, $n=5$ mice per group; ${ }^{A} P<0.05$ vs. Control basal by MannWhitney $U$ test). MCAo, middle cerebral artery occlusion.

The depletion of PVM was confirmed in vivo 24 hours after the i.c.v. injection of TRITC-Dextran (Figure 7, E-J). To assess the harmlessness of CLO injection on microglial cell numbers, we used CX3CR1$\mathrm{GFP}^{+/-}$mice in which TRITC-Dextran ${ }^{+} \mathrm{PVM}$ can be distinguished in vivo from $\mathrm{GFP}^{+}$microglial cells, since PVM are very low-positive for GFP (Figure 7, E and G). PVM depletion did not alter microglial cell numbers $\left(\mathrm{GFP}^{+}\right.$cells, Figure $\left.7 \mathrm{~J}\right)$. The phagocytosis of TRITC-Dextran by PVM was confirmed by IHC; the TRITC-Dextran ${ }^{+}$cells were costained with CD206 (Figure 7F). PVM depletion was confirmed in vivo by 2 -photon imaging (Figure 7, E and H; $P<0.01$ vs. PBS) and by IHC analyses (CD206 staining; Figure 7, G and I; $P<0.01$ vs. PBS).

The specific depletion of PVM by CLO prevented the aggravating effect of chronic alcohol exposure on ischemic lesions (Figure 7, K and L; $P<0.05$ vs. PBS; $n=5-6$ mice/group), whereas CLO treatment did not modify ischemic lesions in control mice (not exposed to alcohol) (Figure 7, $\mathrm{K}$ and L; $n=5-6$ mice/group).

In order to explore the mechanisms of the beneficial effect of PVM depletion on stroke in alcohol-exposed mice, we studied the immune responses triggered 24 hours after stroke ( $n=4$ mice/group). We analyzed microglial/macrophage cell numbers (Iba1 ${ }^{+}$staining; Figure $8, \mathrm{~A}$ and $\mathrm{B}$ ) and neutrophil infiltration (Figure 8, C and D) in PBS- and CLO-injected mice (PVM-depleted) exposed or not to alcohol. Microglial/ macrophage numbers (Figure 8B), P-selectin protein levels (Figure 8, E and F), and VCAM1 protein levels (Figure 8, G and $\mathrm{H}$ ) were significantly increased after stroke in PBS alcohol-exposed mice compared with PBS-control mice (Figures $8, \mathrm{~A}$ and $\mathrm{B} ; P<0.05$ vs. PBS-treated control mice; $n=4$ mice/group).

The effects of PVM depletion were different between control and alcohol-exposed mice. PVM-depleted control mice showed significantly more microglia/macrophages (Figure 8B; $P<0.05$ vs. PBS control; $n=4$ mice/group), as well as more neutrophils (Figure 8D; $P<0.05$ vs. naive PBS; $n=4$ mice/group) and increased VCAM1 protein levels (Figure $8 \mathrm{H}, P<0.05$ vs. naive PBS; $n=4$ mice/group) in the ipsilateral hemisphere compared with PBS-treated control mice. By contrast, in PVM-depleted alcohol-exposed mice, microglia/macrophage (Figure 8B) and neutrophil (Figure 8D) infiltration — as well as VCAM levels (Figure 8H) — remained unchanged. We only found a significant decrease in P-selectin protein levels between PBS- and CLO-treated alcohol-exposed mice (Figure 8, E and F; $P<0.05$ vs. PBS alcohol-exposed mice; $n=4$ mice/group).

Interestingly, by intravital 2-photon microscopy, we observed that the number of adherent leukocytes was significantly decreased in PVM-depleted mice, independently of alcohol exposure (Figure 8, I, J, L, and M; $P<0.05$ vs. PBS-treated mice; $n=4$ mice/group). This effect was specific of leukocyte adhesion, since no difference was detected in the number of rolling or circulating leukocytes in any of the groups (Figure 8, $\mathrm{K}$ and $\mathrm{N}$ ).

\section{Discussion}

We have performed here a translational study to investigate the effects of chronic alcohol consumption on ischemic stroke outcome. Our clinical results show that HD ( $\geq 6$ drinks/day in the last 5 years) is independently associated (a) to END, (b) to stroke severity baseline, and (c) to higher infarct volume in stroke patients. The baseline characteristics of the study population, and outcomes at 3 months, were similar to those of large multicenter registries, suggesting good external validity of our results (25).

Our results show that the inflammatory response is associated with the risk of END in HD stroke patients. In order to elucidate the role of inflammation on the deleterious effect of HD after stroke, we 
A

In vivo microglial response after systemic inflammation

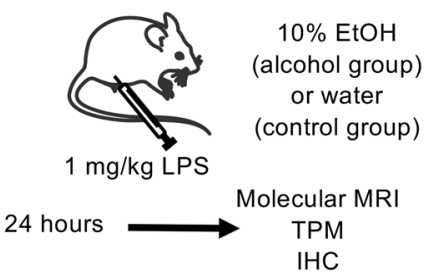

E

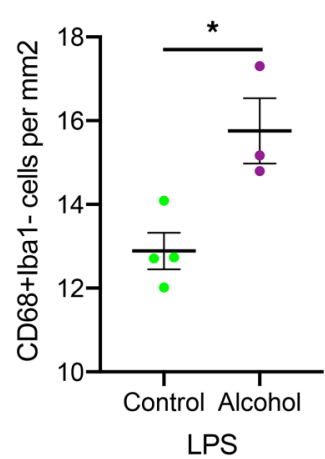

H
Control+LPS ip

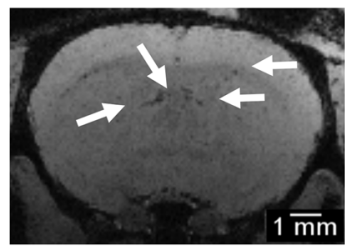

Alcohol+LPS ip

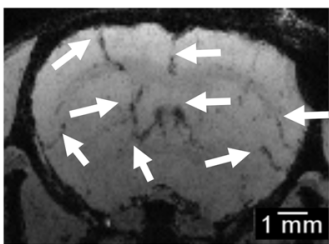

$\mathbf{L}$

K

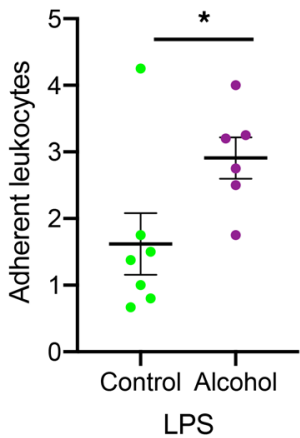

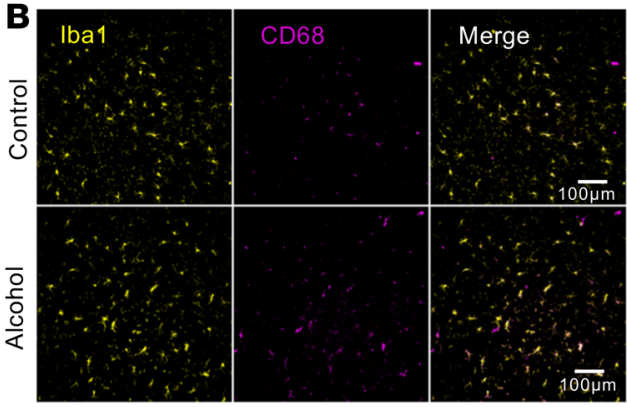

F

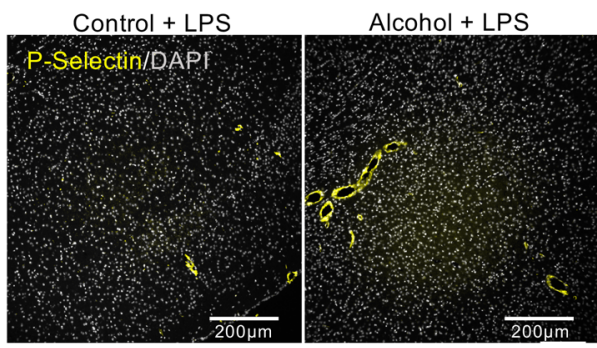

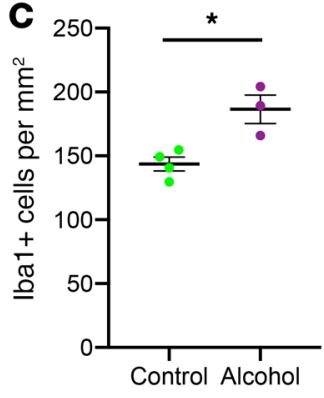

LPS

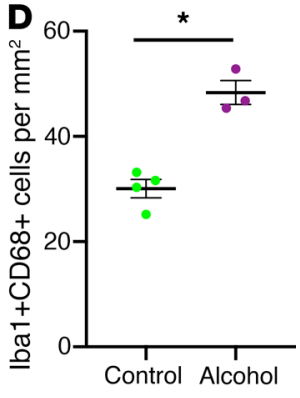

LPS
G
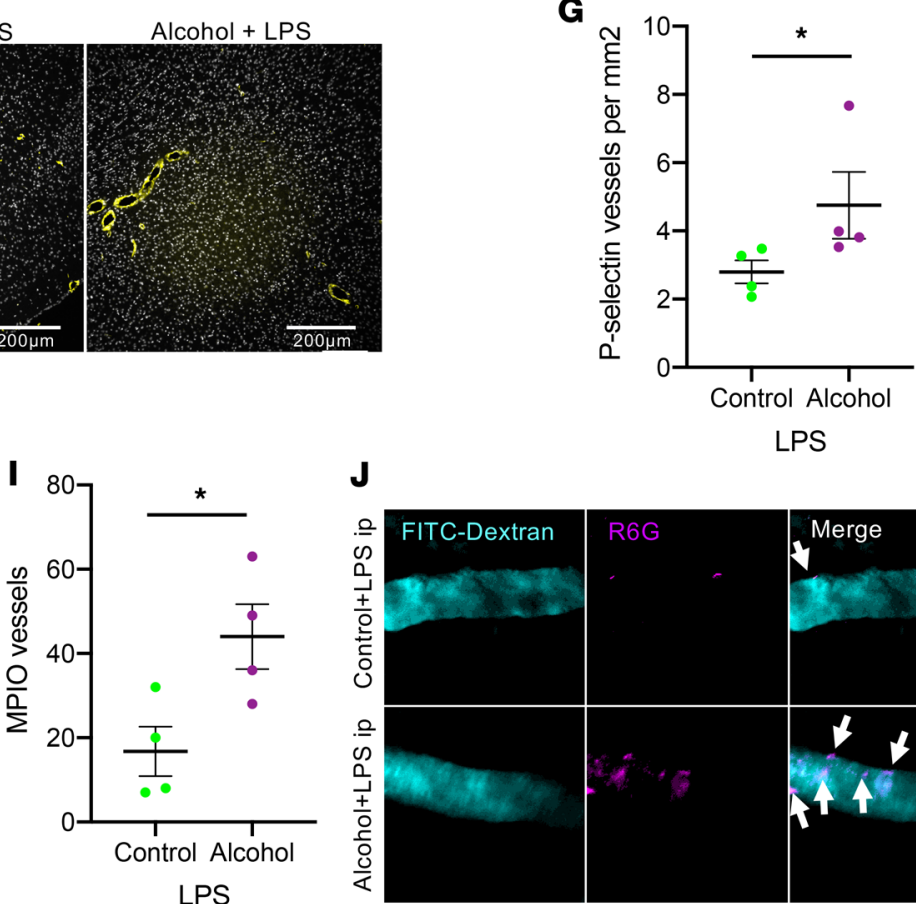

J

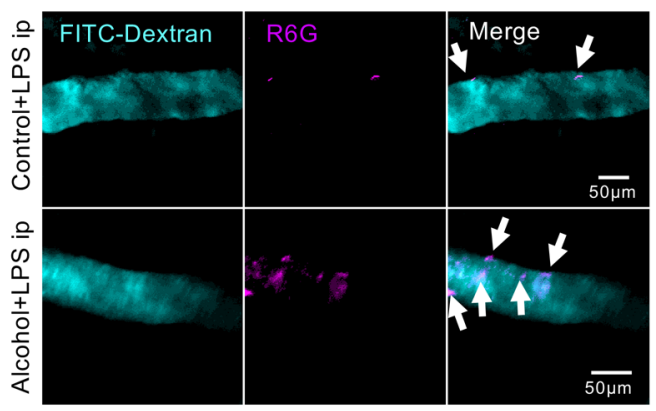

M

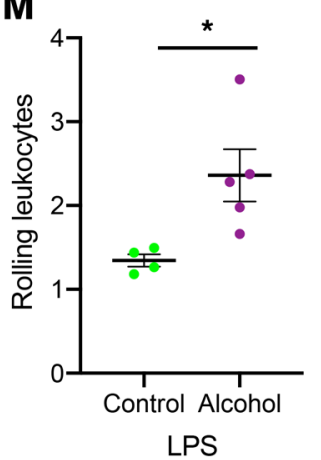

Figure 4. Alcohol exposure exacerbates brain neurovascular inflammatory reactions after an acute systemic insult in mice. (A) Experimental design to study whether chronic alcohol exposure provokes exacerbated inflammatory responses after a secondary injury (peripheral acute LPS injection). (B) Representative photomicrographs of the brain cortex of control and alcohol-exposed mice 24 hours after the acute systemic injection of LPS. Scale bars: $100 \mu \mathrm{m}$. $n=5-6$ mice per group. (C) Quantification of total microglia (Iba1 ${ }^{+}$cells). (D) Quantification of activated microglia (Iba1 ${ }^{+} / \mathrm{CD}^{+}{ }^{+}$cells). (E) Quantification of macrophages (CD68 ${ }^{+} / \mathrm{Iba}^{-}$cells). (F) Representative photomicrographs of P-selectin staining in control and alcohol-exposed mice 24 hours after the acute systemic injection of LPS. Scale bars: $200 \mu \mathrm{m}$. (G) Quantification of the number of P-selectin+ blood vessels. (H) Representative T2*-weighted images showing P-selectin-coupled MPIO in vivo accumulation in the brain of control and alcohol-exposed mice after the acute injection of LPS. Arrows show positive MPIO signals. (I) Quantification of MPIO + blood vessels. (J) Compilation of time-lapse images showing representative in vivo leukocyte adhesion (arrows) obtained by 2-photon microscopy. Scale bars: $50 \mu \mathrm{m}$. (K) Quantification of leukocyte adhesion. (L) Representative time-lapse images of in vivo leukocyte rolling (see also Supplemental Videos 1 and 2 ). Scale bars: $50 \mu$ m.

(M) Quantification of rolling leukocytes per second. ${ }^{*} P<0.05$ versus control, Mann-Whitney $U$ test. 
A

Thromboembolic
ischemic stroke

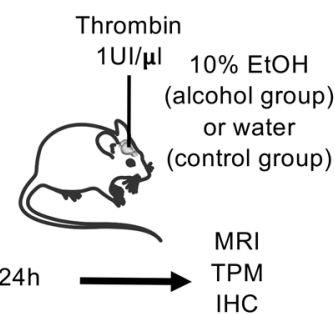

$\mathrm{IHC}$
B

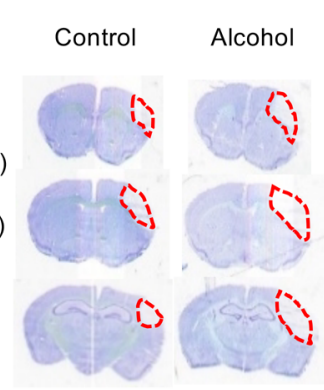

C

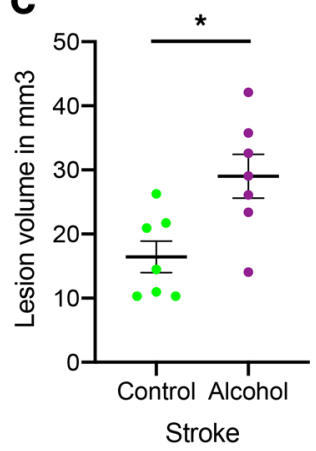

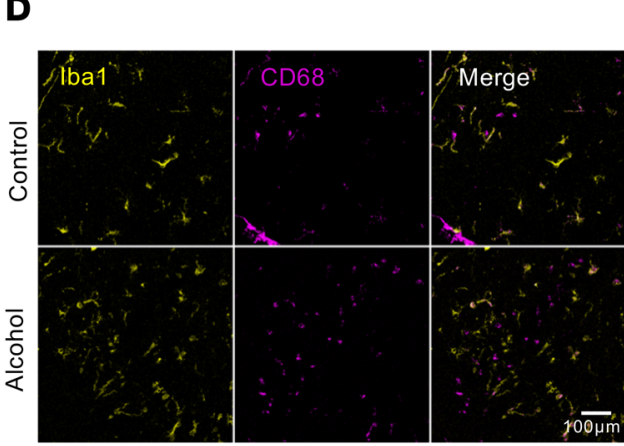

E

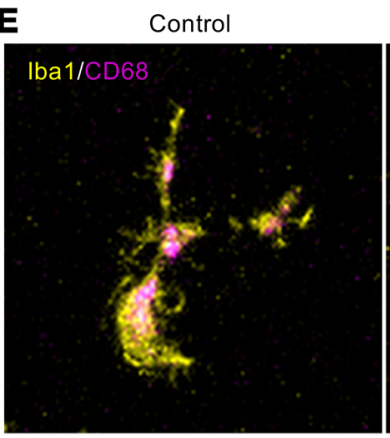

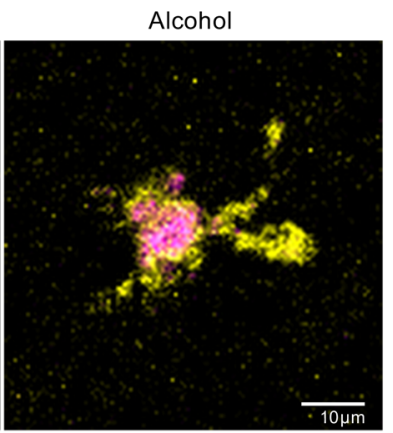

H

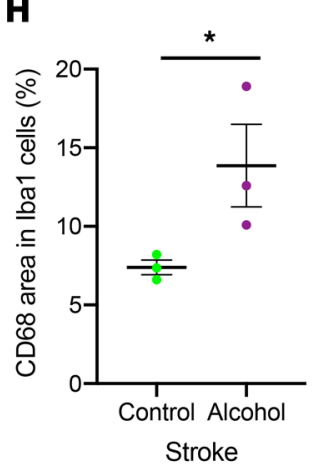

I

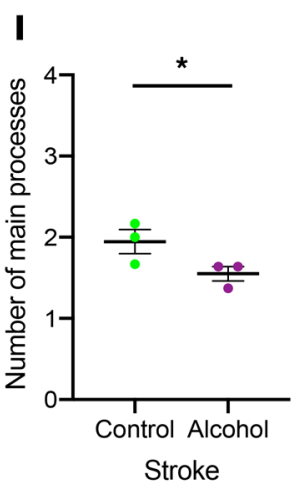

J

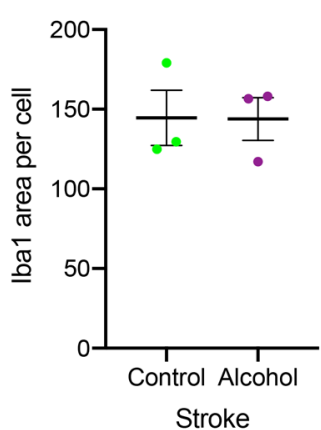

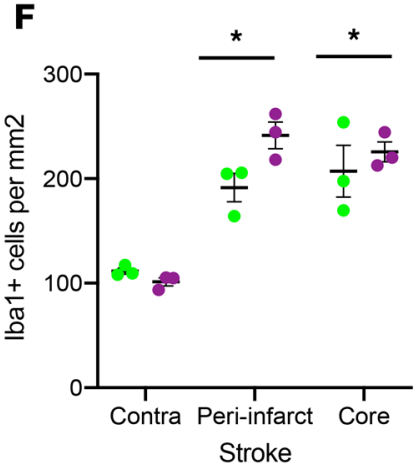

G

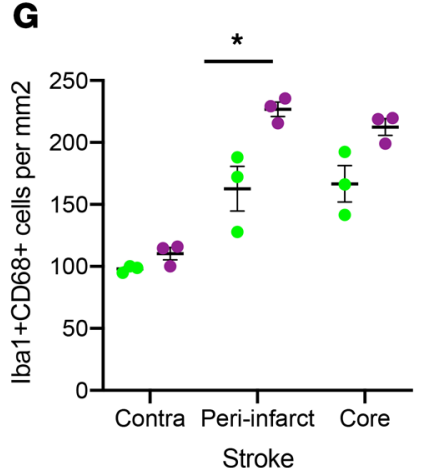

Figure 5. Alcohol exposure increases lesion volume and parenchymal inflammatory responses after ischemic stroke in mice. (A) Experimental design to study whether chronic alcohol exposure worsens ischemic stroke outcome. (B and $\mathbf{C}$ ) Representative brain lesions (B) and corresponding quantifications (C) in control and alcohol-exposed mice 24 hours after stroke onset ( $n=8$ mice/group; ${ }^{*}<0.05$ vs. control mice). (D) Representative photomicrographs of microglial cells and infiltrated macrophages in the ipsilateral cortex 24 hours after stroke onset. Scale bar: $100 \mu \mathrm{m}$. $n=4$ mice per group. (E) High-magnification photomicrographs of microglial cells at the peri-infarct area. Scale bar: $10 \mu \mathrm{m}$. (F) Quantification of Iba1+ cells. (G) Quantification of Iba1+CD68+. (H) Quantification of CD68 area in Iba1 ${ }^{+}$cells. (I) Quantification of the number of processes starting from the soma. (J) Quantification of the mean whole cell area of Iba1+ cells. (K) Quantification of CD68 ${ }^{+}$Iba1 ${ }^{-}$cells. ${ }^{*} P<0.05$. Mann-Whitney $U$ test.

performed preclinical experiments in mice exposed to $10 \%$ ethanol in drinking water during 6 weeks. Our preclinical findings show that chronic alcohol consumption by itself provokes a neurovascular inflammatory priming. The term "priming" is used to describe the propensity of a particular cell type to make an exaggerated response to a secondary stimulus (26) such as intracerebral or systemic LPS injection (27). Microglial priming was first described in the ME7 model of prion disease (28) but has been replicated in other models of chronic neuroinflammatory pathologies, including Alzheimer's disease (29) and Parkinson's disease (30).

To our knowledge, this is the first study defining a neurovascular inflammatory priming induced by chronic alcohol consumption. This alcohol-driven priming affects the brain parenchyma, as well as the perivascular and vascular compartments. In the parenchyma, it is characterized by (a) an unconventional activation profile of microglial cells, in accordance with results described by Cruz et al. (31), and (b) an increase in the expression of TGF- $\beta$ mRNA not accompanied by changes in proinflammatory 
A
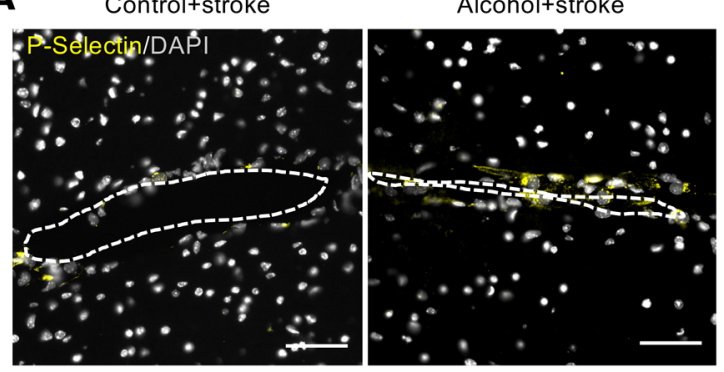

D
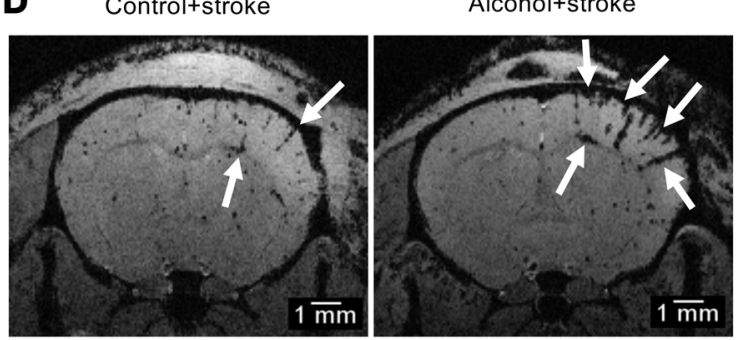

B
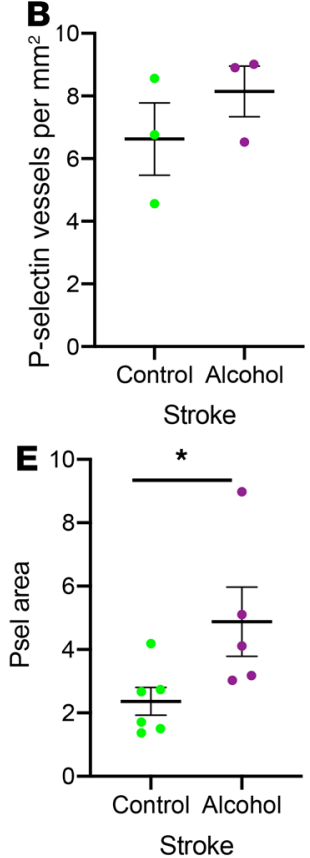

H
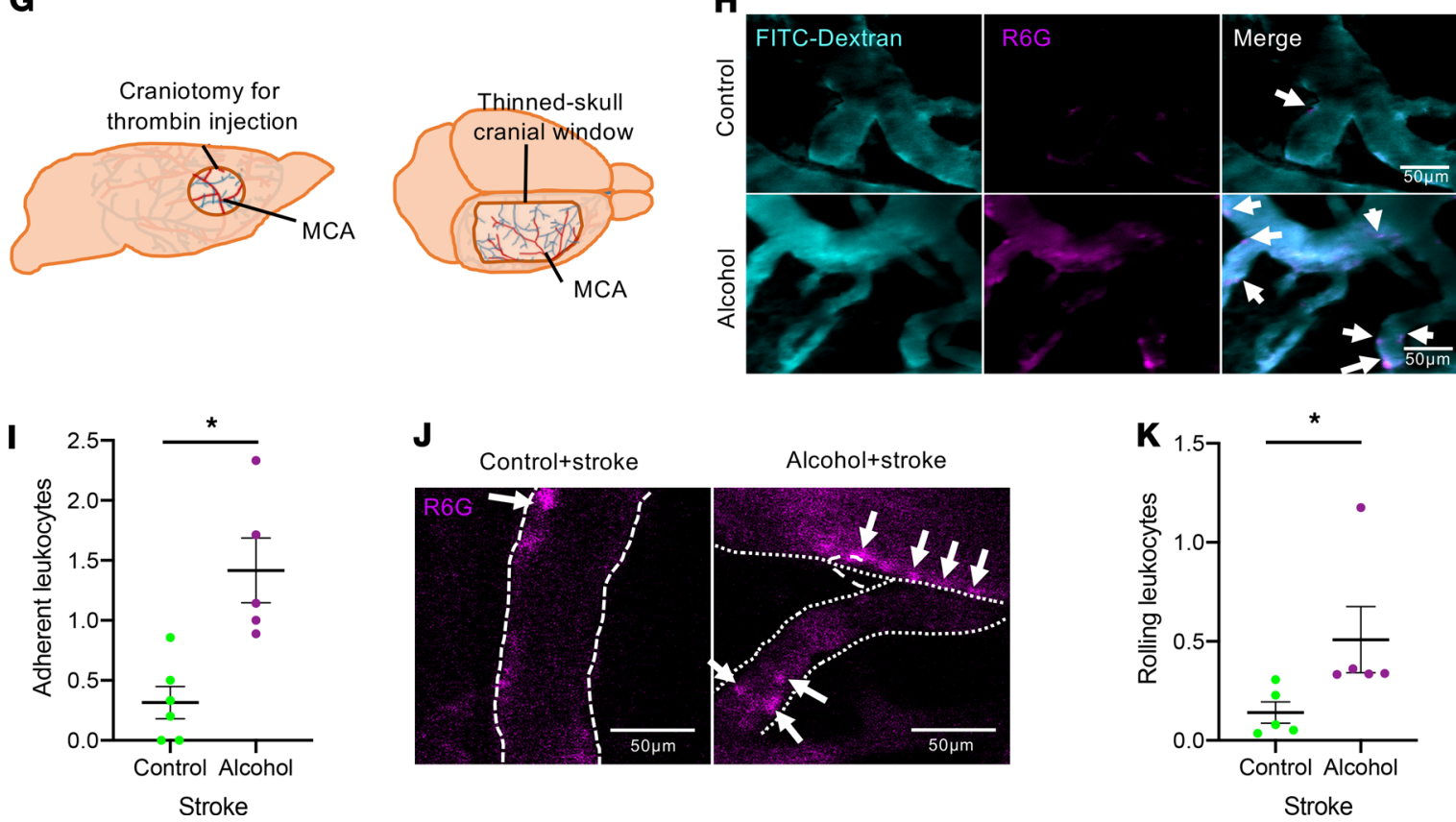

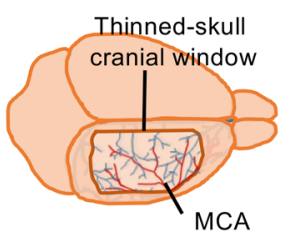

$\mathbf{J}$

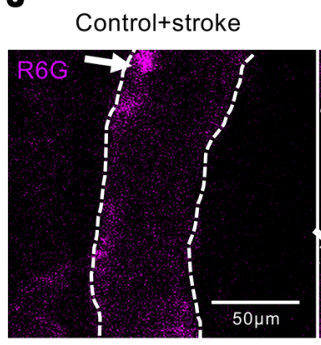

Alcohol+stroke

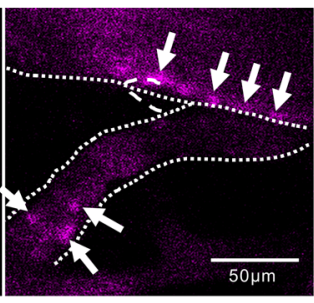

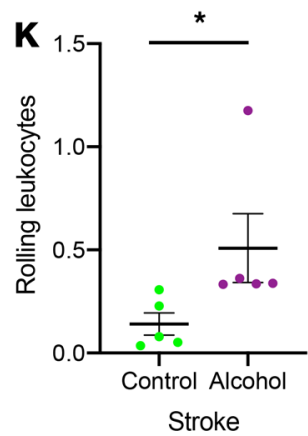

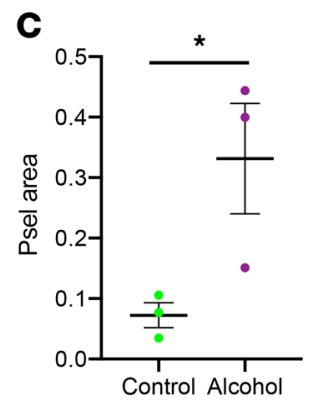

Peri-infarct

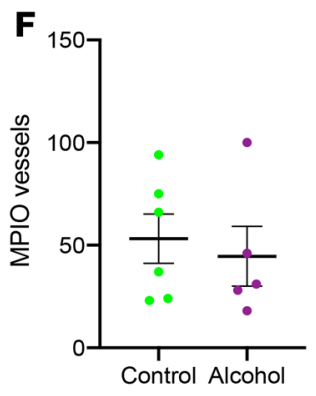

Stroke

Figure 6. Alcohol exposure increases vascular inflammatory responses after ischemic stroke in mice. (A) Representative photomicrographs of P-selectin immunostaining in control and alcohol-exposed mice 24 hours after stroke onset. Scale bars: $50 \mu \mathrm{m}$. (B) Quantification of the number of P-selectin ${ }^{+}$vessels. (C) Quantification of P-selectin+ immunostaining area. (D) Representative T2*-weighted images of in vivo P-selectin molecular imaging. Arrows show MPIO+ blood vessels ( $n=5-6$ mice/group). (E) Quantification of MPIO+ area. (F) Quantification of MPIO+ blood vessels. (G) Schematic view of the craniotomy performed for thrombin injection, which leads to the occlusion of the MCA and the thinned-skull window for intravital 2-photon imaging. (H) Compilation of representative time-lapse images showing in vivo leukocyte adhesion (arrows) obtained by 2-photon microscopy. Scale bars: $50 \mu \mathrm{m}$. (I) Quantification of leukocyte adhesion. (J) Representative time-lapse images of in vivo leukocyte rolling. Scale bars: $50 \mu \mathrm{m}$. (K) Quantification of leukocyte rolling (see also Supplemental Videos 3 and 4 ). $n=4$ mice per group. ${ }^{*} P<0.05$ versus control, Mann-Whitney $U$ test.

cytokines, thus following a similar profile as in priming-driving diseases such as prion disease (32). At the brain perivascular compartment, alcohol provokes an increase in the number of PVM. Finally, at the vascular level, alcohol-induced priming is characterized by increased levels of P-selectin at the endothelial surface, accompanied by an increase in the number of adherent and rolling leukocytes in the brain blood vessels. 
A

In vivo chronic alcohol exposure in mice

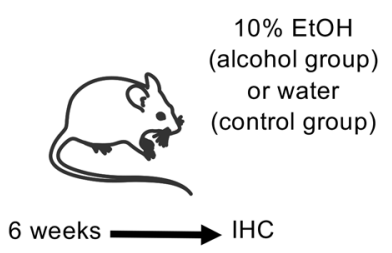

D

In vivo depletion of perivascular macrophages

$10 \mu \mathrm{I}$ PBS/CLO

liposomes (icv)
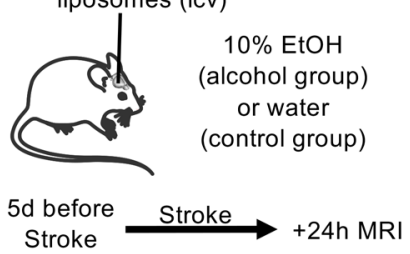

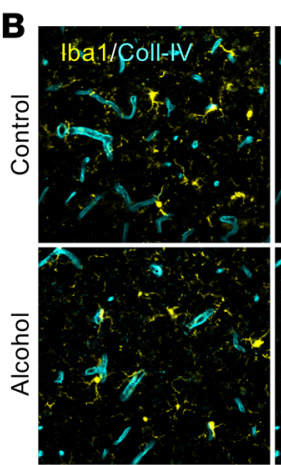

E

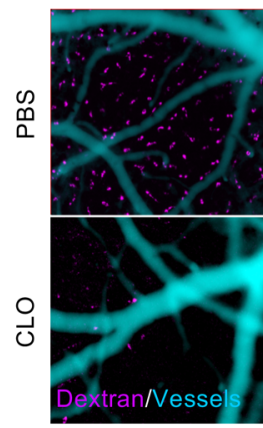

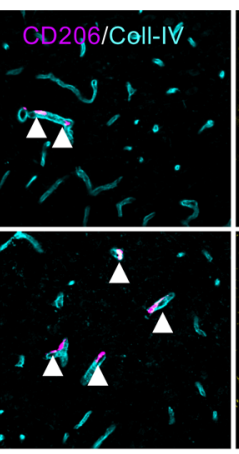

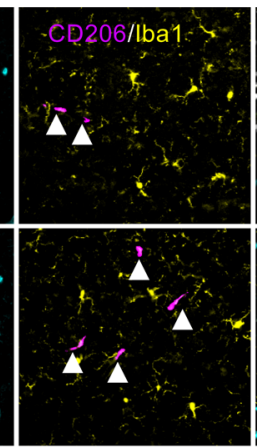

PVM Depletion (2-photon microscopy)

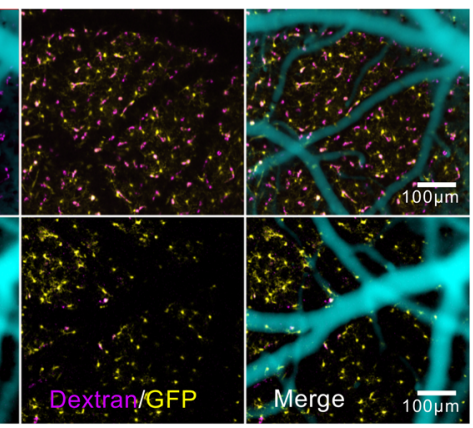

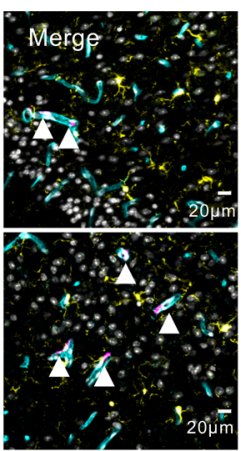

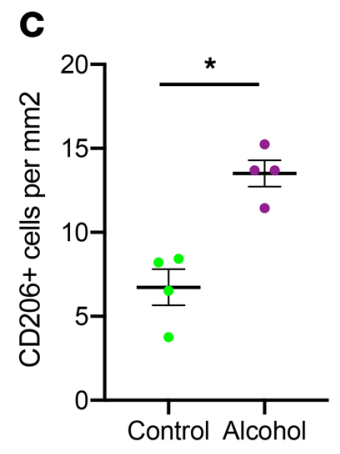

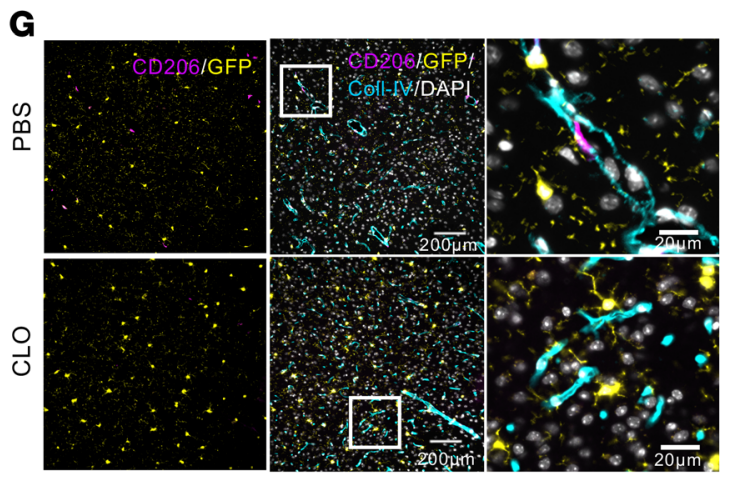

H

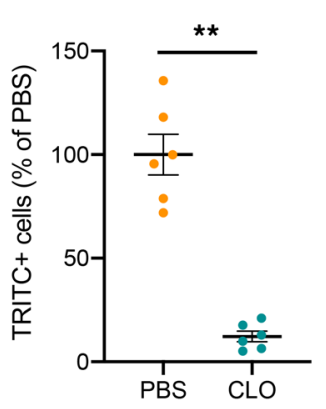

F Epifluorescence microscopy

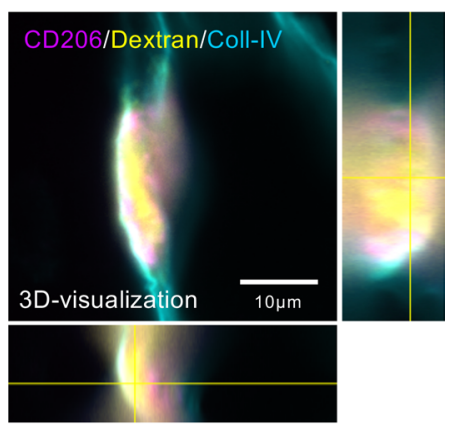

I
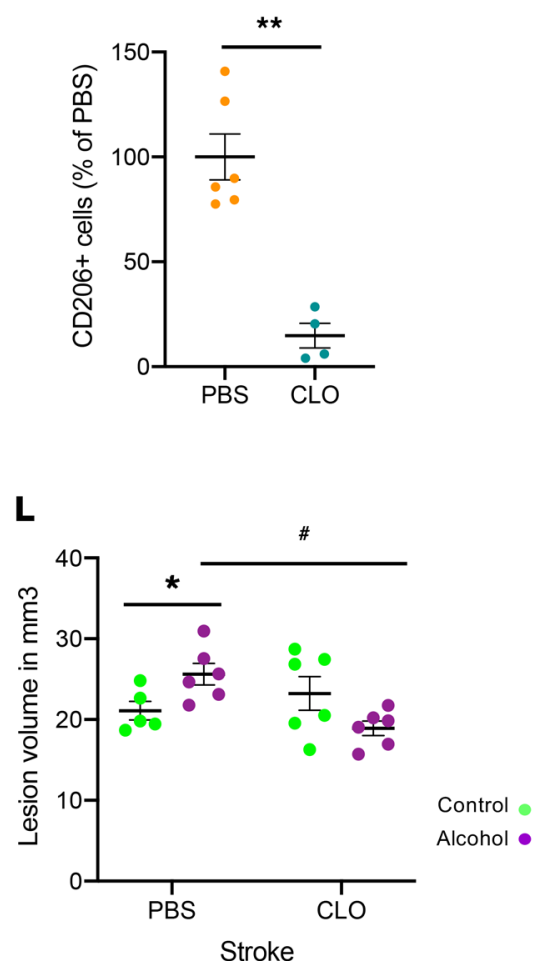

Figure 7. Perivascular macrophages (PVM) mediate the aggravating effect of chronic alcohol consumption on ischemic stroke in mice. (A) Experimental design to study the effects of chronic alcohol exposure on PVM. (B) Representative photomicrographs of PVM CD206+lba1- (arrowheads) cells in control and alcohol-exposed mice. (C) Quantification of PVM (CD206+cells). Scale bars: $20 \mu \mathrm{m}$. (D) Experimental design to deplete PVM in control and alcohol-exposed mice. (E) Representative in vivo Z stack of CX3CR1 ${ }^{\mathrm{CFP+}+-}$ PBS/CLO-injected mice. Blood vessels are visualized through the i.v. injection of FITC-Dextran. Twenty-four hours after the i.c.v. injection of TRITC-Dextran, PVM can be in vivo visualized (note the absence of TRITC-Dextran signals in CLO-treated mice). Microglia are positive for GFP in CX3CR1 ${ }^{\mathrm{CP}+/-}$ mice. Scale bar: $100 \mu \mathrm{m}$. (F) Colocalization of CD206 and phagocyted TRITC-Dextran in PVM (epifluorescence microscopy). Three-dimensional visualization of a double-positive TRITC-Dextran and CD206 PVM for. Scale bar: $10 \mu \mathrm{m}$. (G) Representative photomicrographs of GFP+ microglial cells and CD206 PVMs in PBS- and CLO-treated mice. 
Scale bar: $20 \mu \mathrm{m}$. (H and I) Quantification of PVM depletion by TRITC+ cell counting (H) and CD206+ counting (I). (J) The number of microglial cells remained unchanged after CLO treatment. (K) Representative T2-weighted MRI images showing ischemic lesions in PBS- and CLO-treated naive and alcohol-exposed mice 24 hours after stroke onset. (L) Quantification of lesion volumes. $n=5-6$ mice per group; ${ }^{*} P<0.01,{ }^{*} P<0.05$ versus PBS; ${ }^{~} P<0.05$ versus control. Mann-Whitney $U$ test.

We demonstrate here that alcohol provokes a brain inflammatory priming in mice by using 2 different approaches. First, we intracortically injected inert latex beads to control and alcohol-exposed mice. Eight hours after the injection, alcohol-exposed mice showed significantly more phagocyted beads than control mice, thus demonstrating that chronic alcohol exposure makes microglia more prone to phagocytosis. Second, we systemically injected a single dose of LPS to control and alcohol-exposed mice and studied the subsequent parenchymal, perivascular, and vascular inflammatory responses 24 hours later. In accordance with our priming hypothesis, mice exposed to alcohol showed increased total microglial and activated microglial cell numbers; increased levels of P-selectin in the brain vasculature, characteristic of increased endothelial activation; and significantly increased rolling and adhering leukocyte numbers after the injection of LPS. Previous studies have reported that the exposure of C57BL/6J mice to 10 daily doses of ethanol followed by a LPS or Poly I:C challenge results in a sustained increase of proinflammatory cytokines in the brain compared with control-challenged animals $(33,34)$.

In accordance with our inflammatory priming hypothesis, our data show that inflammatory responses at the parenchymal, perivascular, and vascular levels were exacerbated in alcohol-exposed mice also after stroke. More precisely, microglial numbers and phagocytic capacity, brain PVM/infiltrated macrophages numbers, P-selectin levels at the brain endothelium, and leukocyte rolling and adhesion to brain vasculature were all exacerbated in alcohol-exposed mice after stroke. Inflammatory responses participate in the progression of ischemic lesions (35), but the causality or consequence relationship between the exacerbation of inflammatory responses and the increased lesion volume is difficult to determine. For this reason, our data on the inflammatory status before the ischemic injury are crucial and show that, even in the absence of stroke, HD alters the inflammatory status in both humans and mice. It has been proposed that inflammatory priming could have significant implications for acute sterile inflammatory insults, such as stroke and traumatic brain injury occurring on a background of aging or neurodegeneration (26). Our data show that chronic HD triggered a similar generalized heightened inflammatory sensitivity than in the aforementioned models of neurodegenerative diseases, and that alcohol-induced inflammatory priming had extremely deleterious consequences in stroke, not only in mice, but also in humans.

Importantly, we show here that PVM are determinant for the exacerbation of ischemic lesions, since their specific depletion by CLO blocks the aggravating effect of chronic alcohol exposure on stroke. Interestingly, the beneficial effect of PVM depletion on lesion volume is exclusive of alcohol-exposed mice, in which PVM numbers are significantly increased. Indeed, PVM accumulation at the perivascular space seems to be deleterious for ischemic stroke outcome, as it has been described in other neurological pathologies such as multiple sclerosis or Alzheimer's disease (for review, see ref. 20). It is also possible that alcohol exposure provokes not only an increase in PVM numbers, but also a shift of PVM phenotype and function from a scavenger, "buffer" cell $(16,17)$ to a proinflammatory, ROS-producing cell in "primed" conditions (36). In accordance with this hypothesis, it has recently been described that homeostatic subsets of CNS endogenous tissue macrophages are able to quickly change their phenotypes and generate context- and time-dependent subsets (37). An additional question that deserves future studies is whether PVM could drive microglial priming during chronic alcohol exposure, or instead, if microglial cells are responsible for the accumulation of PVM, at the perivascular spaces and the activation of brain endothelial cells.

Concerning the hypothesis of a role of PVM on mediating the stroke-induced inflammatory responses, our data show that, in naive mice, PVM do modulate stroke-induced inflammatory responses such as microglial activation/macrophage infiltration, neutrophil infiltration, endothelial activation, and leukocyte rolling/adhesion in vivo. It is important to take into account the effects of CLO, per se, found in our study, especially concerning lymphoid cells, which could also modify the post-stroke inflammatory responses. Surprisingly, these altered inflammatory responses are not associated with changes in the final lesion volume between PBS- and CLO-treated naive mice. These data are in accordance with the results reported by Pedragosa et al. (38), in which modifications of granulocyte infiltration in PVM-depleted mice are observed but are not accompanied by changes in final lesion volume in a model of ischemia/reperfusion. These results are in agreement with previous studies showing 
A
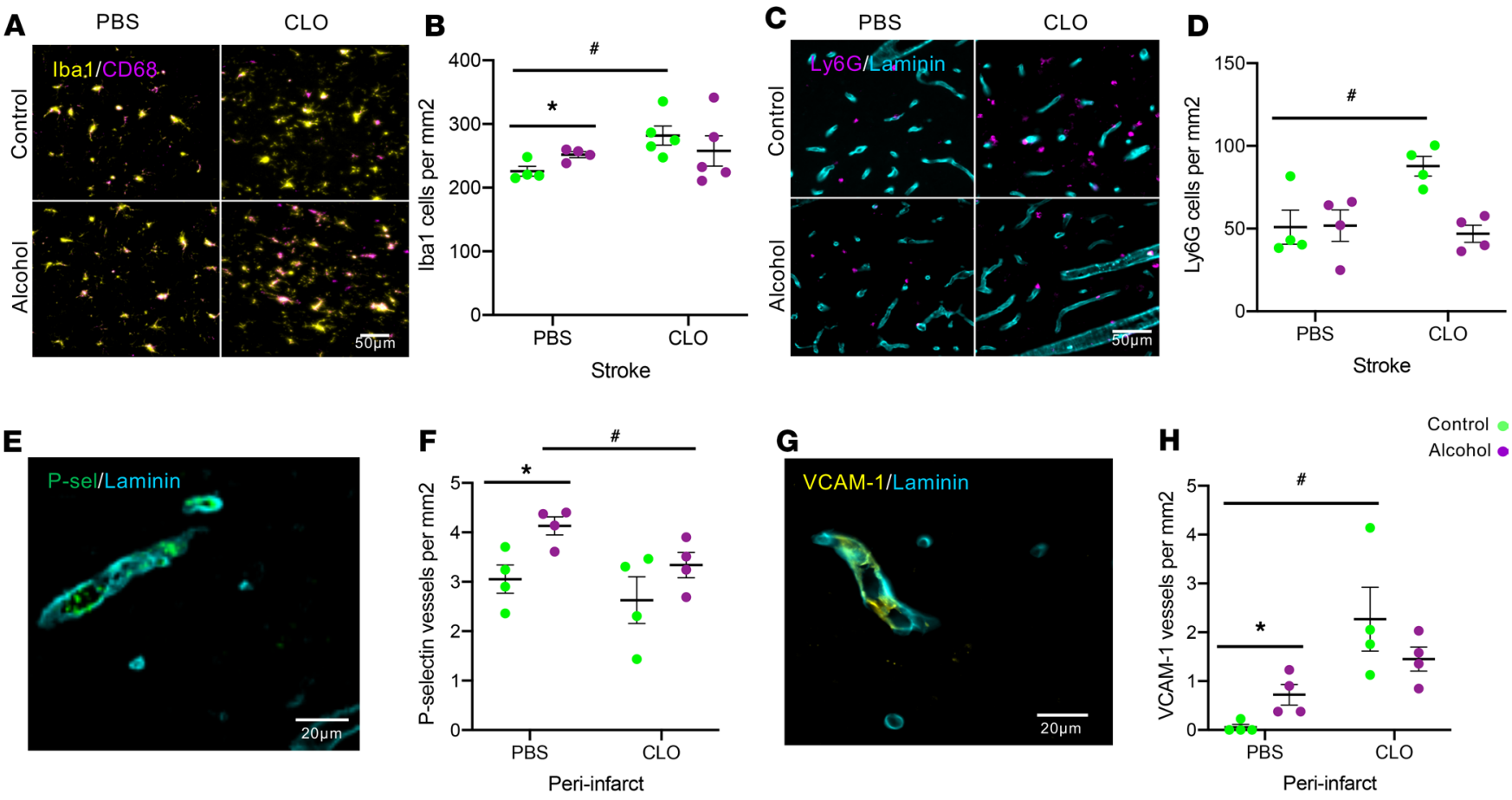
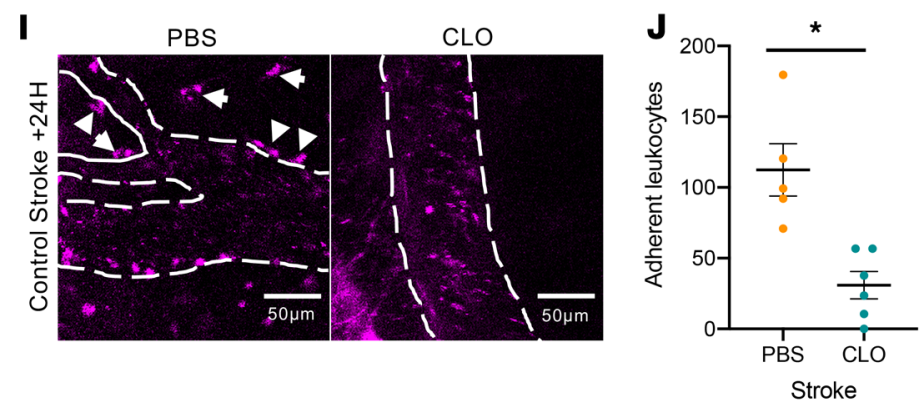

$\mathbf{L}$

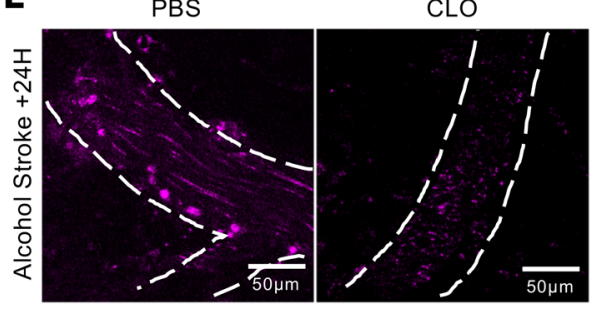

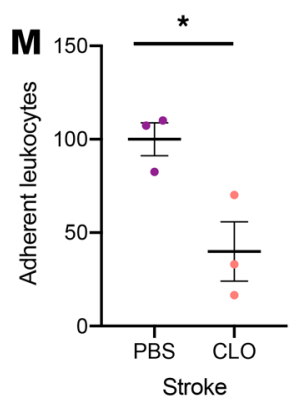
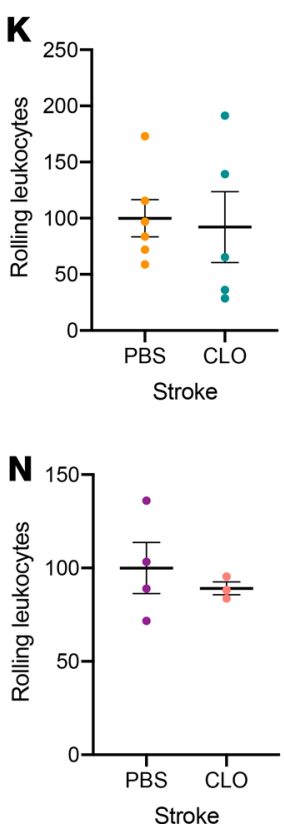

Figure 8. PVM modulate inflammatory responses to stroke in naive mice but not in alcohol-exposed mice. (A) Representative photomicrographs of different subsets of microglia/macrophages 24 hours after stroke onset at the core of the lesion. Scale bar: $50 \mu \mathrm{m}$. (B) Quantification of Iba1 ${ }^{+}$cells. (C) Representative photomicrographs of Ly6 $\mathrm{G}^{+}$neutrophils at the core of the lesion. Scale bar: $50 \mu \mathrm{m}$. (D) Quantification of neutrophil numbers. (E) Representative photomicrographs of P-selectin staining 24 hours after stroke onset. Scale bar: $20 \mu \mathrm{m}$. (F) Quantification of P-selectin ${ }^{+}$blood vessels. (G) Representative photomicrograph of VCAM1+ blood vessel 24 hours after stroke onset. Scale bar: $20 \mu \mathrm{m}$. (H) Quantification of VCAM1+ blood vessels. (I) Representative photomicrographs obtained by 2-photon microscopy of leukocytes (in magenta, Rhodamine-6C) in PBS- and CLO-treated mice (not exposed to alcohol) 24 hours after stroke onset. Scale bars: $50 \mu \mathrm{m}$. (J) Quantification of adherent leukocytes. (K) Quantification of circulating/rolling leukocytes. (L) Representative photomicrographs obtained by 2-photon microscopy of leukocytes (in magenta, Rhodamine-6G) in PBS- and CLO-treated mice (exposed to alcohol) 24 hours after stroke onset. Scale bars: $50 \mu \mathrm{m}$. (M) Quantification of adherent leukocytes. (N) Quantification of circulating/rolling leukocytes. $n=4$ mice per group, ${ }^{*} P<0.05$ versus PBS; $\# \mathrm{P}<0.05$ versus control PBS (B, D, H) and versus alcohol PBS (F). Mann-Whitney $U$ test. 
no impact of leukocyte recruitment on ischemic stroke volume $(39,40)$. Further studies are needed to better understand the links between final lesion volume and leukocyte infiltration at the ischemic area.

Our data suggest that the previous inflammatory status is critical for the ischemic stroke outcome. In the context of alcohol-induced inflammatory priming, PVM are linked to the aggravating effects of alcohol on stroke lesion volumes, since PVM-depleted mice show smaller lesion volumes. This aggravating effect of alcohol in lesion volume is associated with an increased inflammatory response in which PVM seem to play a critical role, since PVM-depleted mice show an attenuated inflammatory response compared with alcohol-exposed mice with intact PVM. One possible hypothesis to explain this differential role of PVM upon naive conditions and alcohol exposure could be a phenotypic change in PVM during alcohol exposure, as it has been described for other neuroinflammatory conditions (37), that could be crucial in case of a secondary CNS injury such as ischemic stroke.

Although research on PVM has intensified in recent times, we still have many unanswered questions concerning PVM phenotype and origin in pathological contexts. Also, since CLO depletes both meningeal macrophages and PVM, the observed effects cannot be attributed exclusively to PVM. In any case, PVM seem to be potential therapeutic targets for limiting the aggravating effects of risk factors on stroke outcome.

A potential limitation of this study is that we cannot exclude an impact of alcohol withdrawal on the worsening outcome of $\mathrm{HD}$ stroke patients and mice. Alcohol-withdrawn neurons are more sensitive to excitotoxic injuries (41) characteristic of early phases of ischemic stroke. Indeed, we cannot exclude that this mechanism also participates to the aggravation of stroke lesions observed in our study, making the exacerbated inflammatory responses observed after stroke the consequence and not the cause of the aggravated stroke outcome. However, our preclinical data obtained on mice not subjected to alcohol withdrawal (the intracortical latex bead-injected mice and the systemic LPS-injected mice) demonstrate that the exacerbated inflammatory response to both latex beads and acute LPS is present even in the absence of alcohol withdrawal. Other mechanisms that could contribute to the aggravation of stroke severity could be an endothelial dysfunction induced by chronic alcohol consumption $(42,43)$, which could have an impact by itself on microglial priming and PVM accumulation.

Interestingly, our data and the recently published data on the beneficial effect of low-dose alcohol consumption on inflammation following transient focal cerebral ischemia in rats (7) suggest that, similarly to the biphasic effects of alcohol on the risk of ischemic stroke (2), alcohol consumption also could have a biphasic effect on the consequences of ischemic stroke. These data, thus, help to clarify the apparently controversial clinical results found on the impact of alcohol consumption on stroke $(4,5)$.

In conclusion, we show that chronic alcohol consumption provokes, by itself, a neurovascular inflammatory priming in mice and inflammatory responses in HD patients. We experimentally demonstrate that this priming, notably involving PVM, drives the exacerbation of the damages provoked by a secondary insult such as an ischemic stroke. Our study opens potentially new avenues for the study of strategies targeting the alcohol-induced inflammatory priming before any neurological insult, in order to prevent a worsened neurological outcome in heavy drinkers.

\section{Methods}

Stroke cohort study population and patient characteristics. We retrospectively analyzed a cohort of 3,645 ischemic stroke patients from the stroke registry of the Stroke Unit of the Neurology Department of the University Clinical Hospital of Santiago de Compostela (Spain) included from January 2010 to December 2016. Acute management (diagnostic and treatment) of patients with stroke was performed according the protocol described by the European Stroke Organization.

Stroke outcome variables. To evaluate the influence of chronic alcohol consumption on the outcome of stroke patients, the following primary outcomes variables were considered: (a) neurological stroke severity determined by the NIHSS at admission, 24 hours, and 48 hours; (b) END; (c) infarct volume determined by CT scan between the 4th and 7th day after admission; (d) degree of disability at 3 months assessed by modified Rankin scale (mRS); and (e) percentage of patients with good outcome at 3 months (mRS $\leq 2)$.

To determine the association between chronic alcohol consumption and inflammatory response, the following biological variables were included in the analysis: (a) leukocyte numbers, (b) fibrinogen, (c) $\mathrm{CRP}$, and (d) sedimentation rate. Axillary temperature over $37.5^{\circ} \mathrm{C}$ at admission was also considered as a marker of hyperthermia.

History of arterial hypertension was considered when the blood pressure was more than 140/90 $\mathrm{mmHg}$ at least 2 different days before stroke onset, if the patient was diagnosed for hypertension, or when 
the patient was under antihypertensive treatment. History of diabetes disease was defined as serum glucose levels of 7.0 or more mmol/L, if the patient was diagnosed of diabetes, or when the patients was under diabetic medication. Smoking patients were defined as those patients who presented smoking habits in the last 5 years. HD habits were defined as a daily alcohol consumption of 6 or more drinks/day in the last 5 years.

Stroke cohort statistical analyses are detailed in the Supplementary Methods.

HD patients without stroke. We retrospectively analyzed data from an independent cohort including 34 patients with a DSM-5 (Diagnostic and Statistical Manual of Mental Disorders, 5th edition) diagnostic of severe Alcohol Use Disorder (AUD) (HD patients) and 21 healthy control subjects. All the participants were informed about the study, approved by the local ethics committee (CPP Nord Ouest III, IDRCB: 2011-A00495-36), and provided their written informed consent before their inclusion.

Blood samples were collected from fasted participants, either at inclusion (control subjects) or the day after admission to hospital (HD patients). Immune cell counts (leukocytes, neutrophils, eosinophils, basophils, lymphocytes, and monocytes) were measured in all participants. CRP levels were measured only in HD patients.

Additional information about this cohort and statistical analyses are detailed in the Supplemental Methods.

Experimental study design. The goal of this study was to investigate the impact of HD on stroke outcome and describe the underlying mechanisms in an experimental model of ischemic stroke in mice.

Animals were randomized to treatment groups, and all analyses were performed by investigators blinded to group allocation. Unblinding was performed after completion of statistical analysis. All animal experiments were performed and reported in accordance with the Animal Research: Reporting of In Vivo Experiments (ARRIVE) guidelines (http://www.nc3rs.org.uk).

Animals. Two-month-old male Swiss mice (35-45 g) (Centre Universitaire de Ressources Biologiques, Normandy University, Caen, France) were housed at $21^{\circ} \mathrm{C}$ in a 12 -hour light/dark cycle with food and water (control group) or a 10\% (v/v) alcohol solution (alcohol group) with ad libitum access for 6 weeks. All mice were checked daily for fluid consumption, health, and abnormal behavior. The average daily liquid intake and weight gain were similar between both groups ( $\sim 6 \mathrm{~mL}$ of liquid intake/mouse/day and a weight gain of $\sim 6 \mathrm{~g}$ between the beginning and the end of the alcohol exposure period; final weight $\sim 40 \mathrm{~g}$ in both groups). Blood alcohol levels (BAL) were measured at the pharmacology unit of Caen University Hospital (Caen, France) in mice at the end of the 6 weeks of alcohol exposure ( $n=10$ mice). At the moment of the blood extraction, only 3 of 10 mice showed positive BAL values $(0.39,0.54$, and $0.63 \mathrm{~g} / \mathrm{L})$; the rest of the mice showed nondetectable values $(<0.1 \mathrm{~g} / \mathrm{L})$.

For intravital 2-photon imaging of PVM, male C57BL/6J CX3CR1-GFP ${ }^{+/-}$mice (The Jackson Laboratory and in-house) were used ( $n=6$ mice/group).

All the procedures needing anesthesia of the mice were performed by an initial exposure to $5 \%$ isoflurane, followed by a maintaining phase of $1.5 \%-2 \%$ isoflurane $30 \% \mathrm{O}_{2} / 70 \% \mathrm{~N}_{2} \mathrm{O}$.

Thromboembolic focal cerebral ischemia. We used the in situ thromboembolic stroke model consisting of the injection of thrombin directly into the middle cerebral artery as described before (9). In order to mimic clinical conditions, alcohol solutions were changed by water after stroke onset and until killing. Additional information is provided in the Supplementary Methods.

I.p. LPS injection. A subset of control and alcohol-exposed mice ( $n=5-6$ mice per group) were i.p. injected with a single dose of the bacterial endotoxin LPS (1 mg/kg) (Sigma-Aldrich) (44) and underwent 2-photon imaging and molecular MRI 24 hours after the injection of LPS. Alcohol-exposed mice maintained free access to the alcohol solution after LPS injection and until killing.

In vivo microglial phagocytic capacity measurement. The protocol consisted of the injection of $1 \mu \mathrm{L}$ of nonionic latex beads in the brain cortex and was modified from Hughes et al. (45). Details are provided in the Supplemental Methods.

Vascular adhesion molecular imaging. MPIOs (diameter $1.08 \mathrm{~mm}$ ) (Invitrogen) covalently conjugated to purified polyclonal goat anti-mouse antibodies for P-selectin (R\&D Systems, clone AF737) were prepared as previously described (46). Details are provided in the Supplemental Methods.

In vivo detection of BBB leakage. Three-dimensional T1 FLASH sequences (spatial resolution $70 \mathrm{~mm} \times 70$ $\mathrm{mm}$; Echo time/repetition time (TE/TR) 4.46/15; 3 averages; 4 minutes, 2 seconds) were used before and 15 minutes after the i.v. injection of $200 \mu \mathrm{L}$ of a solution containing $50 \mu \mathrm{L}$ of Gadolinium chelate (DOTAREM) diluted in saline, as previously described (47). Additional information is provided in the Supplemental Methods.

Near-infrared detection of BBB leakage. NIRF imaging experiments were performed using a PhotonIMAGER (Biospace), as previously described (48). Additional information is provided in the Supplemental Methods. 
Depletion of PVM. A total of $10 \mu \mathrm{L}$ of PBS-liposomes (PBS group) or CLO-encapsulated liposomes (CLO group) were injected in the left lateral ventricle. Additional information is provided in the Supplemental Methods.

In order to minimize the proinflammatory effects of CLO, per se, the i.c.v. injection of CLO was performed 5 days before the stroke.

In vivo macrophage labeling for the follow-up study of macrophage accumulation in the brain. A total of $200 \mu \mathrm{L}$ of $2 \mathrm{mg} \mathrm{Fe} / \mathrm{kg}$ of nude MPIOs were injected i.v. to naive mice and T2*-w acquisitions were performed at different times after its injection to detect peripheral macrophage accumulation at the brain perivascular spaces. Additional details are provided in the Supplemental Methods.

Intravital 2-photon microscopy. Intravital 2-photon microscopy was performed through a thin-skull cranial window to measure leukocyte rolling and adhesion, as well as PVM visualization. Details are provided in the Supplemental Methods.

Flow cytometry analysis. Five days after the i.c.v. injection of PBS or CLO, mice were deeply anesthetized with isoflurane $5 \%$ and intracardially perfused with $1 \times$ PBS (Sigma-Aldrich). Cell isolation from brains (without the cerebellum) and flow cytometry acquisition were performed as previously described (47) to obtain 2 separate panels for myeloid cells or lymphoid cells (see Supplemental Table 6 and Supplemental Figure 5A). Data were analyzed with the FlowJo 7.6.5 software (TreeStar Inc.). Data are expressed as total cell count for each sample.

Quantitative PCR analyses. Details on the methods for quantitative PCR (qPCR) analyses are provided in the Supplemental Methods. Primers used for this study are detailed in Supplemental Table 5.

$I H C$. Epifluorescence and confocal microscopy analyses were performed in brain sections and isolated brain vessels. Details on the protocol and methods are provided in the Supplemental Methods.

Statistics. Results are the mean \pm SEM. Statistical analyses were performed by the Mann-Whitney $U$ test using the Statview software.

Study approval. Stroke patient registry was approved by the Ethics Committee of Galicia (CEIC) (Spain, protocol code 201/516). Signed informed consent was obtained from patients or a relative before study inclusion. Experimental studies were approved by the French ministry of education and research (Project 3748; Center agreement D14118001). All the participants were informed about the study, approved by the local ethics committee (CPP Nord Ouest III, IDRCB 2011-A00495-36) and provided their written informed consent before their inclusion. All animal experiments were performed and reported in accordance with the Animal Research: Reporting of In Vivo Experiments (ARRIVE) guidelines (http://www.nc3rs.org.uk), in accordance with French laws (act no. 87-848; Ministère de 1'Agriculture et de la Forêt) and European Commmunities Council Dorectives of November 24, 1986 (86/609/EEC) guidelines, and they have been approved by the ethical committee (affiliation 3748).

\section{Author contributions}

MR designed and coordinated the study and was responsible for stroke model surgeries. AD and DL performed histological and transcriptional analyses, as well as in vivo functional analyses of microglial phagocytosis. AD, DL, AQ, and EL were responsible for molecular MRI. AD and MR were responsible for 2-photon microscopy. AD, DL, and MN analyzed immunohistochemical samples. MG and MR analyzed flow cytometry. AL and ALP analyzed data from the ALCOBRAIN cohort of HD patients. FC and JC analyzed data and writing of results from the stroke cohort of patients. DV supervised the study. MR wrote the manuscript with the revision and approval of all authors.

\section{Acknowledgments}

The authors are grateful to Carine Ali and Fabian Docagne for their valuable scientific suggestions, as well as Laurent Coulbaut for the analyses of human samples from the ALCOBRAIN cohort. The authors thank Véronique Lelong-Boulouard for the BAL measurements. This study was funded by the Fondation pour la Recherche en Alcoologie (MR), the AXA Research Found (MR), INSERM, Caen-Normandy University, the Regional Council of Normandy, the ANR grant RHU MARVELOUS (ANR-16-RHUS-0009) (DV), and ANR "Retour jeune chercheur" (ALP).

Address correspondence to: Marina Rubio, PHIND Boulevard Henri Becquerel 14074 Caen Cedex, Caen, France. Phone: 33.2.31.47.01.55; Email: rubio@cyceron.fr. 
1. Rehm J, et al. Defining substance use disorders: do we really need more than heavy use? Alcohol Alcohol. 2013;48(6):633-640.

2. Larsson SC, Wallin A, Wolk A, Markus HS. Differing association of alcohol consumption with different stroke types: a systematic review and meta-analysis. BMC Med. 2016;14(1):178.

3. Kadlecová P, Andel R, Mikulík R, Handing EP, Pedersen NL. Alcohol consumption at midlife and risk of stroke during 43 years of follow-up: cohort and twin analyses. Stroke. 2015;46(3):627-633.

4. Ducroquet A, et al. Influence of chronic ethanol consumption on the neurological severity in patients with acute cerebral ischemia. Stroke. 2013;44(8):2324-2326.

5. Gattringer T, et al. IV thrombolysis in patients with ischemic stroke and alcohol abuse. Neurology. 2015;85(18):1592-1597.

6. Zhao H, Mayhan WG, Arrick DM, Xiong W, Sun H. Dose-related influence of chronic alcohol consumption on cerebral ischemia/reperfusion injury. Alcohol Clin Exp Res. 2011;35(7):1265-1269.

7. McCarter KD, et al. Effect of Low-Dose Alcohol Consumption on Inflammation Following Transient Focal Cerebral Ischemia in Rats. Sci Rep. 2017;7(1):12547.

8. Lemarchand E, et al. Impact of alcohol consumption on the outcome of ischemic stroke and thrombolysis: role of the hepatic clearance of tissue-type plasminogen activator. Stroke. 2015;46(6):1641-1650.

9. Orset C, et al. Mouse model of in situ thromboembolic stroke and reperfusion. Stroke. 2007;38(10):2771-2778.

10. Orset C, et al. Efficacy of Alteplase in a Mouse Model of Acute Ischemic Stroke: A Retrospective Pooled Analysis. Stroke. 2016;47(5):1312-1318.

11. Imhof A, Froehlich M, Brenner H, Boeing H, Pepys MB, Koenig W. Effect of alcohol consumption on systemic markers of inflammation. Lancet. 2001;357(9258):763-767.

12. Alho H, Sillanaukee P, Kalela A, Jaakkola O, Laine S, Nikkari ST. Alcohol misuse increases serum antibodies to oxidized LDL and C-reactive protein. Alcohol Alcohol. 2004;39(4):312-315.

13. He J, Crews FT. Increased MCP-1 and microglia in various regions of the human alcoholic brain. Exp Neurol. 2008;210(2):349-358.

14. Goldmann T, et al. Origin, fate and dynamics of macrophages at central nervous system interfaces. Nat Immunol. 2016;17(7):797-805

15. He H, et al. Perivascular Macrophages Limit Permeability. Arterioscler Thromb Vasc Biol. 2016;36(11):2203-2212.

16. Kida S, Steart PV, Zhang ET, Weller RO. Perivascular cells act as scavengers in the cerebral perivascular spaces and remain distinct from pericytes, microglia and macrophages. Acta Neuropathol. 1993;85(6):646-652.

17. Mendes-Jorge L, et al. Scavenger function of resident autofluorescent perivascular macrophages and their contribution to the maintenance of the blood-retinal barrier. Invest Ophthalmol Vis Sci. 2009;50(12):5997-6005.

18. Mato $\mathrm{M}$, et al. Involvement of specific macrophage-lineage cells surrounding arterioles in barrier and scavenger function in brain cortex. Proc Natl Acad Sci USA. 1996;93(8):3269-3274.

19. Fabriek BO, et al. CD163-positive perivascular macrophages in the human CNS express molecules for antigen recognition and presentation. Glia. 2005;51(4):297-305.

20. Faraco G, Park L, Anrather J, Iadecola C. Brain perivascular macrophages: characterization and functional roles in health and disease. J Mol Med. 2017;95(11):1143-1152.

21. Faraco G, et al. Perivascular macrophages mediate the neurovascular and cognitive dysfunction associated with hypertension. J Clin Invest. 2016;126(12):4674-4689.

22. Anrather J, Iadecola C. Inflammation and Stroke: An Overview. Neurotherapeutics. 2016;13(4):661-670.

23. Perego C, Fumagalli S, De Simoni MG. Temporal pattern of expression and colocalization of microglia/macrophage phenotype markers following brain ischemic injury in mice. J Neuroinflammation. 2011;8:174

24. Polfliet MM, Goede PH, van Kesteren-Hendrikx EM, van Rooijen N, Dijkstra CD, van den Berg TK. A method for the selective depletion of perivascular and meningeal macrophages in the central nervous system. J Neuroimmunol. 2001;116(2):188-195.

25. Dávalos A et al. Citicoline in the treatment of acute ischaemic stroke: an international, randomised, multicentre, placebo-controlled study (ICTUS trial). Lancet. 2012;380(9839):349-357.

26. Hennessy E, Griffin ÉW, Cunningham C. Astrocytes Are Primed by Chronic Neurodegeneration to Produce Exaggerated Chemokine and Cell Infiltration Responses to Acute Stimulation with the Cytokines IL-1 $\beta$ and TNF- $\alpha$. J Neurosci. 2015;35(22):8411-8422.

27. Cunningham C, Wilcockson DC, Campion S, Lunnon K, Perry VH. Central and systemic endotoxin challenges exacerbate the local inflammatory response and increase neuronal death during chronic neurodegeneration. J Neurosci. 2005;25(40):9275-9284

28. Perry VH, Cunningham C, Boche D. Atypical inflammation in the central nervous system in prion disease. Curr Opin Neurol. 2002;15(3):349-354.

29. Sly LM, et al. Endogenous brain cytokine mRNA and inflammatory responses to lipopolysaccharide are elevated in the Tg2576 transgenic mouse model of Alzheimer's disease. Brain Res Bull. 2001;56(6):581-588.

30. Pott Godoy MC, Tarelli R, Ferrari CC, Sarchi MI, Pitossi FJ. Central and systemic IL-1 exacerbates neurodegeneration and motor symptoms in a model of Parkinson's disease. Brain. 2008;131(Pt 7):1880-1894.

31. Cruz SA, et al. Loss of IRF2BP2 in Microglia Increases Inflammation and Functional Deficits after Focal Ischemic Brain Injury. Front Cell Neurosci. 2017;11:201.

32. Cunningham C, Boche D, Perry VH. Transforming growth factor beta1, the dominant cytokine in murine prion disease: influence on inflammatory cytokine synthesis and alteration of vascular extracellular matrix. Neuropathol Appl Neurobiol. 2002;28(2):107-119.

33. Qin L, He J, Hanes RN, Pluzarev O, Hong JS, Crews FT. Increased systemic and brain cytokine production and neuroinflammation by endotoxin following ethanol treatment. J Neuroinflammation. 2008;5:10.

34. Qin L, Crews FT. Chronic ethanol increases systemic TLR3 agonist-induced neuroinflammation and neurodegeneration. J Neuroinflammation. 2012;9:130.

35. Iadecola C, Anrather J. The immunology of stroke: from mechanisms to translation. Nat Med. 2011;17(7):796-808.

36. Capone C, Faraco G, Park L, Cao X, Davisson RL, Iadecola C. The cerebrovascular dysfunction induced by slow pressor doses of angiotensin II precedes the development of hypertension. Am J Physiol Heart Circ Physiol. 2011;300(1):H397-H407.

37. Jordão MJC, et al. Single-cell profiling identifies myeloid cell subsets with distinct fates during neuroinflammation. Science. 
2019;363(6425):eaat7554.

38. Pedragosa J, et al. CNS-border associated macrophages respond to acute ischemic stroke attracting granulocytes and promoting vascular leakage. Acta Neuropathol Commun. 2018;6(1):76.

39. Harris AK, Ergul A, Kozak A, Machado LS, Johnson MH, Fagan SC. Effect of neutrophil depletion on gelatinase expression, edema formation and hemorrhagic transformation after focal ischemic stroke. BMC Neurosci. 2005;6:49.

40. Schmidt A, et al. Targeting Different Monocyte/Macrophage Subsets Has No Impact on Outcome in Experimental Stroke. Stroke. 2017;48(4):1061-1069.

41. Rubio M, et al. Pharmacological activation/inhibition of the cannabinoid system affects alcohol withdrawal-induced neuronal hypersensitivity to excitotoxic insults. PLOS ONE. 2011;6(8):e23690.

42. Mayhan WG. Responses of cerebral arterioles during chronic ethanol exposure. Am J Physiol. 1992;262(3 Pt 2):H787-H791.

43. Sun H, Patel KP, Mayhan WG. Tetrahydrobiopterin, a cofactor for NOS, improves endothelial dysfunction during chronic alcohol consumption. Am J Physiol Heart Circ Physiol. 2001;281(5):H1863-H1869.

44. Montagne A, et al. Ultra-sensitive molecular MRI of cerebrovascular cell activation enables early detection of chronic central nervous system disorders. Neuroimage. 2012;63(2):760-770.

45. Hughes MM, Field RH, Perry VH, Murray CL, Cunningham C. Microglia in the degenerating brain are capable of phagocytosis of beads and of apoptotic cells, but do not efficiently remove PrPSc, even upon LPS stimulation. Glia. 2010;58(16):2017-2030.

46. Quenault A, et al. Molecular magnetic resonance imaging discloses endothelial activation after transient ischaemic attack. Brain. 2017;140(1):146-157.

47. Drieu A et al. Immune Responses and Anti-inflammatory Strategies in a Clinically Relevant Model of Thromboembolic Ischemic Stroke with Reperfusion. Transl Stroke Res. September 2019.

48. Marcos-Contreras OA, et al. Hyperfibrinolysis increases blood-brain barrier permeability by a plasmin- and bradykinin-dependent mechanism. Blood. 2016;128(20):2423-2434 